\title{
Guiding Target Synthesis with Statistical Modeling Tools: A Case Study in Organocatalysis
}

\author{
Isaiah O. Betinol, Yutao Kuang and Jolene P. Reid* \\ Correspondence to: jreid@,chem.ubc.ca \\ Department of Chemistry, University of British Columbia, 2036 Main Mall, Vancouver, British \\ Columbia, V6T 1Z1, Canada
}

Table of Contents

Full list of authors in the Gaussian reference

Computational details $\quad 3$

Cartesian coordinates of the reaction components relevant to chiral phosphate catalysis 4

Cartesian coordinates of the reaction components relevant to secondary amine catalysis 37

Models Used 45

Out-of-sample Predictions $\quad 48$

$\begin{array}{ll}\text { Data curation } & 49\end{array}$

Principal Component Analysis (PCA) 50

Analysis of Variance (ANOVA)

Histogram of Observed Error $\quad 56$

$\begin{array}{ll}\text { References } & 58\end{array}$ 


\section{Full list of authors in the Gaussian reference}

M. J. Frisch, G. W. Trucks, H. B. Schlegel, G. E. Scuseria, M. A. Robb, J. R. Cheeseman, G. Scalmani, V. Barone, G. A. Petersson, H. Nakatsuji, X. Li, M. Caricato, A. V. Marenich, J. Bloino, B. G. Janesko, R. Gomperts, B. Mennucci, H. P. Hratchian, J. V. Ortiz, A. F. Izmaylov, J. L. Sonnenberg, D. Williams-Young, F. Ding, F. Lipparini, F. Egidi, J. Goings, B. Peng, A. Petrone, T. Henderson, D. Ranasinghe, V. G. Zakrzewski, J. Gao, N. Rega, G. Zheng, W. Liang, M. Hada, M. Ehara, K. Toyota, R. Fukuda, J. Hasegawa, M. Ishida, T. Nakajima, Y. Honda, O. Kitao, H. Nakai, T. Vreven, K. Throssell, J. A. Montgomery, Jr., J. E. Peralta, F. Ogliaro, M. J. Bearpark, J. J. Heyd, E. N. Brothers, K. N. Kudin, V. N. Staroverov, T. A. Keith, R. Kobayashi, J. Normand, K. Raghavachari, A. P. Rendell, J. C. Burant, S. S. Iyengar, J. Tomasi, M. Cossi, J. M. Millam, M. Klene, C. Adamo, R. Cammi, J. W. Ochterski, R. L. Martin, K. Morokuma, O. Farkas, J. B. Foresman, and D. J. Fox, Gaussian, Inc., Wallingford CT, 2016. 


\section{Computational details}

Unless otherwise noted, all reaction components were optimized in the gas-phase with the M06$2 \mathrm{X}$ density functional, ${ }^{1}$ and the triple- $\zeta$ valence quality def2-TZVP basis set of Weigend and Ahlrichs, ${ }^{2}$ as implemented in Gaussian 16 (revision C.01). ${ }^{3}$ All of the optimized geometries were verified by frequency computations as minima (zero imaginary frequencies). Gaussian input files were written using a Python script. ${ }^{4}$ Parameters were acquired from these ground state structures. NBO charges were calculated using NBO6, ${ }^{5}$ at the same level. Sterimol values were calculated using a modified version of Paton's Python script to accommodate non-terminal reference atoms. ${ }^{6}$

Conformational searches were performed with Macromodel version $11.7^{7}$ and the OPLS4 or OPLS3 force field. ${ }^{8}$

Multidimensional regression analyses were performed using MATLAB ${ }^{\circledR} .9$ 


\section{Cartesian coordinates of the reaction components relevant to chiral phosphate catalysis}

The following reaction components were optimized with Gaussian 16 (rev. C.01). All other reaction components required for re-creating the statistical model have been published elsewhere. ${ }^{10}$<smiles>C=C(NC(=O)OCc1ccccc1)c1ccccc1</smiles>

\section{Enamide 1}

M06-2X/def2-TZVP Geometry

$\begin{array}{lrrr}\mathrm{C} & 1.71231 & 0.95164 & -0.23019 \\ \mathrm{C} & 1.86055 & 2.02445 & 0.64368 \\ \mathrm{C} & 0.93523 & 3.05815 & 0.65069 \\ \mathrm{C} & -0.15154 & 3.02700 & -0.21185 \\ \mathrm{C} & -0.31106 & 1.95447 & -1.07988 \\ \mathrm{C} & 0.61145 & 0.92191 & -1.08563 \\ \mathrm{H} & 2.69174 & 2.03254 & 1.33747 \\ \mathrm{H} & 1.05782 & 3.88361 & 1.34005 \\ \mathrm{H} & -0.87847 & 3.82893 & -0.20036 \\ \mathrm{H} & -1.16426 & 1.91320 & -1.74427 \\ \mathrm{H} & 0.47650 & 0.08309 & -1.75791 \\ \mathrm{C} & 2.72122 & -0.13485 & -0.26527 \\ \mathrm{C} & 4.02841 & 0.08584 & -0.14311 \\ \mathrm{~N} & 2.24943 & -1.43204 & -0.56693 \\ \mathrm{C} & 1.14538 & -2.04855 & -0.04764 \\ \mathrm{H} & 2.76562 & -1.99685 & -1.22110 \\ \mathrm{H} & 4.73808 & -0.73025 & -0.15926 \\ \mathrm{H} & 4.40916 & 1.09266 & -0.05672 \\ \mathrm{O} & 0.69513 & -3.08098 & -0.48070 \\ \mathrm{O} & 0.66134 & -1.37642 & 1.00461 \\ \mathrm{C} & -0.64430 & -1.75793 & 1.46368 \\ \mathrm{H} & -0.76664 & -2.83117 & 1.32821 \\ \mathrm{H} & -0.64779 & -1.51689 & 2.52440 \\ \mathrm{C} & -3.61342 & 0.49744 & -0.65660 \\ \mathrm{C} & -3.09116 & -0.66355 & -1.21375 \\ \mathrm{C} & -2.14388 & -1.40345 & -0.52104 \\ \mathrm{C} & -1.70429 & -0.98189 & 0.73234 \\ \mathrm{C} & -2.22513 & 0.18463 & 1.27939 \\ \mathrm{C} & -3.17943 & 0.92102 & 0.59132 \\ \mathrm{H} & -4.35920 & 1.06891 & -1.19447 \\ \mathrm{H} & -3.43073 & -0.99848 & -2.18551 \\ \mathrm{H} & -1.73494 & -2.31217 & -0.94660 \\ & & & \end{array}$




$\begin{array}{llll}\mathrm{H} & -1.87639 & 0.52160 & 2.24867 \\ \mathrm{H} & -3.58034 & 1.82678 & 1.02784\end{array}$<smiles>C=C(NC(=O)OCc1ccccc1)c1ccc(C)cc1</smiles>

Enamide 2

$\begin{array}{lccc}\text { M06-2X/def2-TZVP Geometry } & \\ \mathrm{C} & 1.25234 & 1.42754 & -0.19714 \\ \mathrm{C} & 0.34000 & 0.93195 & -1.12680 \\ \mathrm{C} & -0.97007 & 1.37624 & -1.13411 \\ \mathrm{C} & -1.41583 & 2.31376 & -0.20632 \\ \mathrm{C} & -0.50418 & 2.79680 & 0.72794 \\ \mathrm{C} & 0.81255 & 2.36205 & 0.73463 \\ \mathrm{H} & 0.65767 & 0.18728 & -1.84703 \\ \mathrm{H} & -1.66683 & 0.96932 & -1.85731 \\ \mathrm{C} & -2.84191 & 2.78764 & -0.21812 \\ \mathrm{H} & -0.83399 & 3.51185 & 1.47297 \\ \mathrm{H} & 1.50024 & 2.72364 & 1.48902 \\ \mathrm{C} & 2.66099 & 0.96692 & -0.20947 \\ \mathrm{C} & 3.69645 & 1.77529 & 0.00358 \\ \mathrm{~N} & 2.87649 & -0.38038 & -0.58352 \\ \mathrm{C} & 2.17056 & -1.46371 & -0.14089 \\ \mathrm{H} & 3.57366 & -0.59210 & -1.27788 \\ \mathrm{H} & 4.70994 & 1.39772 & 0.00588 \\ \mathrm{H} & 3.54350 & 2.83435 & 0.15060 \\ \mathrm{O} & 2.21403 & -2.55127 & -0.66151 \\ \mathrm{O} & 1.46340 & -1.16032 & 0.95631 \\ \mathrm{C} & 0.47376 & -2.11301 & 1.36857 \\ \mathrm{H} & 0.40962 & -1.99876 & 2.44866 \\ \mathrm{H} & 0.82207 & -3.11489 & 1.12382 \\ \mathrm{C} & -3.27457 & -1.20720 & -0.50891 \\ \mathrm{C} & -2.97574 & -0.67733 & 0.73842 \\ \mathrm{C} & -1.76474 & -0.97727 & 1.34575 \\ \mathrm{C} & -0.84769 & -1.81215 & 0.71766 \\ \mathrm{C} & -1.15057 & -2.33793 & -0.53611 \\ \mathrm{C} & -2.35866 & -2.03427 & -1.14744 \\ \mathrm{H} & -4.22079 & -0.97827 & -0.98319 \\ \mathrm{H} & -3.68420 & -0.02823 & 1.23753 \\ \mathrm{H} & -1.52605 & -0.55677 & 2.31580 \\ \mathrm{H} & -0.43114 & -2.98319 & -1.02660 \\ \mathrm{H} & -2.59018 & -2.45047 & -2.11956 \\ \mathrm{H} & -2.94441 & 3.69903 & -0.81166\end{array}$




$\begin{array}{lllr}\mathrm{H} & -3.19238 & 3.01048 & 0.79009 \\ \mathrm{H} & -3.49776 & 2.03260 & -0.65241\end{array}$<smiles>C=C(NC(=O)OCc1ccccc1)c1ccc(Cl)cc1</smiles>

Enamide 3

$\begin{array}{lccc}\text { M06-2X/def2-TZVP Geometry } & \\ \mathrm{C} & 0.89379 & -1.67035 & -0.18544 \\ \mathrm{C} & 0.19390 & -0.89253 & -1.10669 \\ \mathrm{C} & -1.18906 & -0.86696 & -1.11030 \\ \mathrm{C} & -1.88029 & -1.61372 & -0.16929 \\ \mathrm{C} & -1.20854 & -2.38445 & 0.76548 \\ \mathrm{C} & 0.17779 & -2.40801 & 0.75181 \\ \mathrm{H} & 0.73622 & -0.29773 & -1.83149 \\ \mathrm{H} & -1.73036 & -0.26030 & -1.82247 \\ \mathrm{Cl} & -3.61312 & -1.55943 & -0.14771 \\ \mathrm{H} & -1.76662 & -2.94733 & 1.50093 \\ \mathrm{H} & 0.71157 & -2.98483 & 1.49630 \\ \mathrm{C} & 2.37547 & -1.71685 & -0.21810 \\ \mathrm{C} & 3.07156 & -2.83291 & -0.01629 \\ \mathrm{~N} & 3.03095 & -0.52569 & -0.60271 \\ \mathrm{C} & 2.74855 & 0.73478 & -0.15124 \\ \mathrm{H} & 3.76353 & -0.56798 & -1.29174 \\ \mathrm{H} & 4.15293 & -2.82786 & -0.03147 \\ \mathrm{H} & 2.56644 & -3.77533 & 0.13622 \\ \mathrm{O} & 3.16607 & 1.73991 & -0.67009 \\ \mathrm{O} & 1.98265 & 0.68993 & 0.94667 \\ \mathrm{C} & 1.37948 & 1.92528 & 1.36286 \\ \mathrm{H} & 1.28938 & 1.84106 & 2.44375 \\ \mathrm{H} & 2.04705 & 2.74718 & 1.11087 \\ \mathrm{C} & -2.46220 & 2.30456 & -0.50359 \\ \mathrm{C} & -1.32697 & 2.78359 & -1.14553 \\ \mathrm{C} & -0.08456 & 2.67690 & -0.53587 \\ \mathrm{C} & 0.03043 & 2.08625 & 0.72011 \\ \mathrm{C} & -1.10984 & 1.60490 & 1.35413 \\ \mathrm{C} & -2.35318 & 1.71507 & 0.74833 \\ \mathrm{H} & -3.43122 & 2.38824 & -0.97903 \\ \mathrm{H} & -1.41058 & 3.24757 & -2.12004 \\ \mathrm{H} & 0.80596 & 3.04738 & -1.03017 \\ \mathrm{H} & -1.02123 & 1.13624 & 2.32757 \\ \mathrm{H} & -3.23439 & 1.33175 & 1.24594\end{array}$


<smiles>C=CC1C(C(N/C=C/OCc2ccc(OC)cc2)c2ccnc3ccc(O)cc23)N2CCN1CC2C=C</smiles>

Enamide 4

$\begin{array}{lccc}\text { M06-2X/def2-TZVP Geometry } & \\ \text { C } & -4.55165 & -0.10783 & 0.36617 \\ \mathrm{C} & -4.08470 & 1.16606 & 0.67602 \\ \mathrm{C} & -3.56708 & 2.00144 & -0.29901 \\ \mathrm{C} & -3.50753 & 1.53591 & -1.61313 \\ \mathrm{C} & -3.94938 & 0.26873 & -1.93615 \\ \mathrm{C} & -4.47041 & -0.55675 & -0.94258 \\ \mathrm{O} & -4.83309 & -1.81939 & -1.33533 \\ \mathrm{C} & -5.68267 & -2.54469 & -0.46764 \\ \mathrm{C} & -3.05181 & 3.37746 & 0.04695 \\ \mathrm{O} & -1.80020 & 3.66690 & -0.55269 \\ \mathrm{C} & -0.80287 & 2.79278 & -0.19113 \\ \mathrm{C} & 0.39702 & 3.24027 & 0.17262 \\ \mathrm{~N} & 1.48389 & 2.44252 & 0.52510 \\ \mathrm{C} & 1.39447 & 1.02496 & 0.25439 \\ \mathrm{C} & 2.80611 & 0.42319 & 0.32965 \\ \mathrm{C} & 0.50639 & 0.31253 & 1.26349 \\ \mathrm{C} & -0.43729 & -0.69299 & 0.90263 \\ \mathrm{C} & -1.11136 & -1.36095 & 1.96206 \\ \mathrm{~N} & -0.92612 & -1.06214 & 3.27260 \\ \mathrm{C} & -0.08882 & -0.10172 & 3.55922 \\ \mathrm{C} & 0.64962 & 0.60756 & 2.59169 \\ \mathrm{C} & -0.77003 & -1.03344 & -0.43320 \\ \mathrm{C} & -1.69008 & -2.00985 & -0.69549 \\ \mathrm{C} & -2.29766 & -2.72895 & 0.36042 \\ \mathrm{C} & -2.02472 & -2.39951 & 1.65292 \\ \mathrm{O} & -2.00373 & -2.29206 & -1.98876 \\ \mathrm{~N} & 3.75749 & 1.18242 & -0.49647 \\ \mathrm{C} & 3.60242 & 0.83557 & -1.91447 \\ \mathrm{C} & 5.11420 & 0.82640 & -0.07634 \\ \mathrm{C} & 2.84429 & -1.07786 & -0.03957 \\ \mathrm{C} & 4.01298 & -0.63531 & -2.17731 \\ \mathrm{C} & 4.13912 & -1.33488 & -0.81944 \\ \mathrm{C} & 5.30800 & -0.71753 & -0.02130 \\ \mathrm{C} & 6.65649 & -1.12536 & -0.52120 \\ \mathrm{C} & 7.56671 & -1.76857 & 0.19564 \\ \mathrm{H} & -4.93242 & -0.74157 & 1.15383\end{array}$




\begin{tabular}{|c|c|c|c|}
\hline $\mathrm{H}$ & -4.11412 & 1.49970 & 1.70678 \\
\hline $\mathrm{H}$ & -3.08644 & 2.17411 & -2.38083 \\
\hline $\mathrm{H}$ & -3.88706 & -0.10954 & -2.94793 \\
\hline $\mathrm{H}$ & -5.96408 & -3.45006 & -0.99980 \\
\hline $\mathrm{H}$ & -5.17335 & -2.81270 & 0.46106 \\
\hline $\mathrm{H}$ & -6.57683 & -1.96274 & -0.23267 \\
\hline $\mathrm{H}$ & -3.72998 & 4.14697 & -0.32536 \\
\hline $\mathrm{H}$ & -2.97690 & 3.47864 & 1.13528 \\
\hline $\mathrm{H}$ & -1.06111 & 1.74154 & -0.24628 \\
\hline $\mathrm{H}$ & 0.57025 & 4.30849 & 0.22175 \\
\hline $\mathrm{H}$ & 2.37656 & 2.80286 & 0.20936 \\
\hline $\mathrm{H}$ & 0.99323 & 0.86315 & -0.75368 \\
\hline $\mathrm{H}$ & 3.14154 & 0.55471 & 1.36299 \\
\hline $\mathrm{H}$ & 0.03559 & 0.13785 & 4.61081 \\
\hline $\mathrm{H}$ & 1.32911 & 1.39392 & 2.89504 \\
\hline $\mathrm{H}$ & -0.33822 & -0.51380 & -1.27696 \\
\hline $\mathrm{H}$ & -2.97926 & -3.53576 & 0.11785 \\
\hline $\mathrm{H}$ & -2.48819 & -2.91586 & 2.48361 \\
\hline $\mathrm{H}$ & -2.93385 & -2.55568 & -2.02724 \\
\hline $\mathrm{H}$ & 2.56531 & 1.01300 & -2.20039 \\
\hline $\mathrm{H}$ & 4.21300 & 1.52638 & -2.49705 \\
\hline $\mathrm{H}$ & 5.81101 & 1.27090 & -0.79131 \\
\hline $\mathrm{H}$ & 5.31837 & 1.27163 & 0.89855 \\
\hline $\mathrm{H}$ & 2.79323 & -1.69392 & 0.85960 \\
\hline $\mathrm{H}$ & 1.98561 & -1.34801 & -0.66073 \\
\hline $\mathrm{H}$ & 3.26391 & -1.14293 & -2.78712 \\
\hline $\mathrm{H}$ & 4.95551 & -0.68576 & -2.72492 \\
\hline $\mathrm{H}$ & 4.30386 & -2.40466 & -0.94983 \\
\hline $\mathrm{H}$ & 5.21825 & -1.05581 & 1.01554 \\
\hline $\mathrm{H}$ & 6.89245 & -0.85236 & -1.54769 \\
\hline $\mathrm{H}$ & 8.53141 & -2.03364 & -0.21674 \\
\hline $\mathrm{H}$ & 7.37447 & -2.05202 & 1.22445 \\
\hline
\end{tabular}

\section{Dienecarbamate 1}

\begin{tabular}{lccc}
\multicolumn{4}{l}{ M06-2X/def2-TZVP Geometry } \\
C & -6.99752 & 1.14866 & 0.01615 \\
C & -5.76766 & 0.53439 & -0.56874 \\
C & -4.60896 & 0.39584 & 0.07451 \\
C & -3.41761 & -0.20201 & -0.49862 \\
C & -2.27452 & -0.32356 & 0.17824 \\
N & -1.11698 & -0.88888 & -0.33952
\end{tabular}




$\begin{array}{lccc}\mathrm{C} & 0.03369 & -0.99872 & 0.38557 \\ \mathrm{O} & 0.17497 & -0.62510 & 1.52189 \\ \mathrm{O} & 0.98224 & -1.59876 & -0.35601 \\ \mathrm{C} & 2.27970 & -1.69912 & 0.25512 \\ \mathrm{C} & 3.07672 & -0.43835 & 0.06752 \\ \mathrm{C} & 3.78141 & -0.23103 & -1.11459 \\ \mathrm{C} & 4.50420 & 0.93636 & -1.30843 \\ \mathrm{C} & 4.52909 & 1.90700 & -0.31569 \\ \mathrm{C} & 3.82980 & 1.70652 & 0.86636 \\ \mathrm{C} & 3.10464 & 0.53868 & 1.05773 \\ \mathrm{H} & -7.82961 & 0.44045 & 0.01600 \\ \mathrm{H} & -6.82505 & 1.47239 & 1.04243 \\ \mathrm{H} & -7.32013 & 2.01503 & -0.56629 \\ \mathrm{H} & -5.83813 & 0.17930 & -1.59404 \\ \mathrm{H} & -4.52807 & 0.74789 & 1.10039 \\ \mathrm{H} & -3.47960 & -0.55901 & -1.52300 \\ \mathrm{H} & -2.16270 & 0.01610 & 1.19975 \\ \mathrm{H} & -1.10178 & -1.23356 & -1.28585 \\ \mathrm{H} & 2.15509 & -1.93326 & 1.31089 \\ \mathrm{H} & 2.75260 & -2.53676 & -0.25361 \\ \mathrm{H} & 3.76361 & -0.99234 & -1.88619 \\ \mathrm{H} & 5.05134 & 1.08742 & -2.22991 \\ \mathrm{H} & 5.09516 & 2.81773 & -0.46293 \\ \mathrm{H} & 3.84795 & 2.46169 & 1.64140 \\ \mathrm{H} & 2.54261 & 0.38187 & 1.96966\end{array}$<smiles>C(=C1/CNCc2ccccc21)\c1ccccc1</smiles>

\section{Indolium 1}

$\begin{array}{lccr}\text { M06-2X/def2-TZVP Geometry } & \\ \mathrm{C} & 3.92829 & 0.85828 & -0.12434 \\ \mathrm{C} & 2.54611 & 0.82080 & -0.15981 \\ \mathrm{C} & 1.79710 & -0.34299 & 0.02723 \\ \mathrm{C} & 2.45755 & -1.54011 & 0.27305 \\ \mathrm{C} & 3.84292 & -1.52702 & 0.30588 \\ \mathrm{C} & 4.56771 & -0.34774 & 0.11146 \\ \mathrm{C} & 0.38904 & 0.03828 & -0.06196 \\ \mathrm{C} & 0.39205 & 1.44057 & -0.32907 \\ \mathrm{~N} & 1.63330 & 1.86813 & -0.38460 \\ \mathrm{C} & -0.70485 & -0.78280 & -0.01759\end{array}$




$\begin{array}{lrrr}\mathrm{C} & -2.09116 & -0.42206 & 0.04732 \\ \mathrm{C} & -3.03364 & -1.33841 & -0.44944 \\ \mathrm{C} & -4.37724 & -1.01953 & -0.46326 \\ \mathrm{C} & -4.80265 & 0.19648 & 0.05945 \\ \mathrm{C} & -3.88661 & 1.09143 & 0.60766 \\ \mathrm{C} & -2.54072 & 0.78875 & 0.60396 \\ \mathrm{H} & 4.48336 & 1.77521 & -0.26985 \\ \mathrm{H} & 1.91433 & -2.46149 & 0.43701 \\ \mathrm{H} & 5.64792 & -0.37619 & 0.14889 \\ \mathrm{H} & -0.43967 & 2.09220 & -0.54622 \\ \mathrm{H} & 1.90013 & 2.81788 & -0.60754 \\ \mathrm{H} & -0.49837 & -1.85010 & -0.05492 \\ \mathrm{H} & -2.69456 & -2.28711 & -0.84708 \\ \mathrm{H} & -5.09620 & -1.71925 & -0.86650 \\ \mathrm{H} & -5.85742 & 0.43829 & 0.06712 \\ \mathrm{H} & -4.23353 & 2.01144 & 1.05797 \\ \mathrm{H} & -1.84044 & 1.44740 & 1.10083 \\ \mathrm{H} & 4.37793 & -2.44874 & 0.48921\end{array}$<smiles>COc1ccc2c(c1)/C(=C/c1ccc(Br)cc1)[NH2+]2</smiles>

\section{Indolium 2}

$\begin{array}{lrrr}\text { M06-2X/def2-TZVP Geometry } & \\ \mathrm{C} & 4.62376 & 1.71211 & -0.14260 \\ \mathrm{C} & 3.26015 & 1.49897 & -0.16892 \\ \mathrm{C} & 2.68794 & 0.22559 & -0.06496 \\ \mathrm{C} & 3.50001 & -0.87982 & 0.07960 \\ \mathrm{C} & 4.88522 & -0.68814 & 0.10081 \\ \mathrm{C} & 5.43727 & 0.59930 & -0.00856 \\ \mathrm{C} & 1.23618 & 0.40929 & -0.11197 \\ \mathrm{C} & 1.03755 & 1.81719 & -0.26635 \\ \mathrm{~N} & 2.20538 & 2.41686 & -0.30040 \\ \mathrm{C} & 0.27898 & -0.56654 & -0.12351 \\ \mathrm{C} & -1.14484 & -0.43019 & -0.02971 \\ \mathrm{C} & -1.94488 & -1.45205 & -0.56843 \\ \mathrm{C} & -3.32013 & -1.35437 & -0.55936 \\ \mathrm{C} & -3.91704 & -0.24516 & 0.03280 \\ \mathrm{C} & -3.14805 & 0.75417 & 0.62894 \\ \mathrm{C} & -1.77429 & 0.66061 & 0.59566 \\ \mathrm{H} & 5.05252 & 2.70230 & -0.22062 \\ \mathrm{H} & 3.11634 & -1.88634 & 0.17931 \\ \mathrm{H} & 6.50776 & 0.73494 & 0.01409\end{array}$




$\begin{array}{lrrr}\mathrm{H} & 0.12048 & 2.36225 & -0.42318 \\ \mathrm{H} & 2.32997 & 3.40935 & -0.44948 \\ \mathrm{H} & 0.64466 & -1.58437 & -0.23847 \\ \mathrm{H} & -1.47351 & -2.31547 & -1.02169 \\ \mathrm{H} & -3.93290 & -2.13101 & -0.99446 \\ \mathrm{Br} & -5.78443 & -0.11092 & 0.06750 \\ \mathrm{H} & -3.63409 & 1.57905 & 1.13068 \\ \mathrm{H} & -1.18362 & 1.39787 & 1.12321 \\ \mathrm{O} & 5.61600 & -1.80170 & 0.23300 \\ \mathrm{C} & 7.03118 & -1.69706 & 0.26510 \\ \mathrm{H} & 7.40081 & -2.71133 & 0.37555 \\ \mathrm{H} & 7.41013 & -1.26862 & -0.66485 \\ \mathrm{H} & 7.35695 & -1.09431 & 1.11510\end{array}$<smiles>COc1ccc2c(c1)/C(=C/c1ccc(C(F)(F)F)cc1)C=N2</smiles>

Indolium 3

$\begin{array}{lccc}\text { M06-2X/def2-TZVP Geometry } & \\ \mathrm{C} & -4.52117 & 1.72867 & -0.16888 \\ \mathrm{C} & -3.16302 & 1.48643 & -0.18479 \\ \mathrm{C} & -2.61738 & 0.20335 & -0.06243 \\ \mathrm{C} & -3.45089 & -0.88304 & 0.09032 \\ \mathrm{C} & -4.83361 & -0.66267 & 0.10249 \\ \mathrm{C} & -5.35831 & 0.63400 & -0.02550 \\ \mathrm{C} & -1.16034 & 0.35770 & -0.10360 \\ \mathrm{C} & -0.93351 & 1.76568 & -0.27314 \\ \mathrm{~N} & -2.08749 & 2.38273 & -0.32077 \\ \mathrm{C} & -0.21785 & -0.62401 & -0.09541 \\ \mathrm{C} & 1.21630 & -0.48110 & -0.01618 \\ \mathrm{C} & 2.01171 & -1.47008 & -0.60783 \\ \mathrm{C} & 3.39084 & -1.35678 & -0.60496 \\ \mathrm{C} & 3.98099 & -0.27457 & 0.02875 \\ \mathrm{C} & 3.20951 & 0.69031 & 0.67113 \\ \mathrm{C} & 1.83493 & 0.58989 & 0.64957 \\ \mathrm{H} & -4.92905 & 2.72641 & -0.26150 \\ \mathrm{H} & -3.08836 & -1.89602 & 0.20291 \\ \mathrm{H} & -6.42588 & 0.79184 & -0.01022 \\ \mathrm{H} & -0.00370 & 2.29071 & -0.42546 \\ \mathrm{H} & -2.19308 & 3.37673 & -0.47893 \\ \mathrm{H} & -0.58641 & -1.64372 & -0.17878 \\ \mathrm{H} & 1.54151 & -2.31681 & -1.09200 \\ \mathrm{H} & 4.00507 & -2.10926 & -1.07917\end{array}$




$\begin{array}{lrrr}\mathrm{C} & 5.48397 & -0.11846 & 0.06040 \\ \mathrm{H} & 3.69377 & 1.49972 & 1.20211 \\ \mathrm{H} & 1.23821 & 1.29704 & 1.21045 \\ \mathrm{O} & -5.58562 & -1.75865 & 0.24442 \\ \mathrm{C} & -6.99956 & -1.62750 & 0.26876 \\ \mathrm{H} & -7.38786 & -2.63344 & 0.38984 \\ \mathrm{H} & -7.31702 & -1.00856 & 1.11007 \\ \mathrm{H} & -7.36567 & -1.20366 & -0.66828 \\ \mathrm{~F} & 5.85126 & 0.98656 & -0.59270 \\ \mathrm{~F} & 5.92104 & -0.00434 & 1.31516 \\ \mathrm{~F} & 6.10327 & -1.15199 & -0.50059\end{array}$<smiles>COc1ccc(/C=C2\C=NCc3ccc(OC)cc32)cc1</smiles>

Indolium 4

$\begin{array}{lrrr}\text { M06-2X/def2-TZVP Geometry } & \\ \mathrm{C} & -3.95911 & 1.64273 & -0.14681 \\ \mathrm{C} & -2.58472 & 1.50160 & -0.16800 \\ \mathrm{C} & -1.94940 & 0.25726 & -0.07042 \\ \mathrm{C} & -2.70808 & -0.89003 & 0.06230 \\ \mathrm{C} & -4.09840 & -0.76935 & 0.07721 \\ \mathrm{C} & -4.71517 & 0.48975 & -0.02566 \\ \mathrm{C} & -0.51118 & 0.51255 & -0.10730 \\ \mathrm{C} & -0.38001 & 1.91934 & -0.24895 \\ \mathrm{~N} & -1.57941 & 2.46958 & -0.28535 \\ \mathrm{C} & 0.48683 & -0.43782 & -0.12049 \\ \mathrm{C} & 1.88877 & -0.28581 & 0.01550 \\ \mathrm{C} & 2.71502 & -1.34377 & -0.41189 \\ \mathrm{C} & 4.08660 & -1.25420 & -0.37147 \\ \mathrm{C} & 4.67886 & -0.09360 & 0.14684 \\ \mathrm{C} & 3.87075 & 0.95320 & 0.63798 \\ \mathrm{C} & 2.51091 & 0.85764 & 0.57301 \\ \mathrm{H} & -4.43763 & 2.61029 & -0.21932 \\ \mathrm{H} & -2.27427 & -1.87634 & 0.15867 \\ \mathrm{H} & -5.79125 & 0.57085 & -0.00758 \\ \mathrm{H} & 0.50777 & 2.51175 & -0.39925 \\ \mathrm{H} & -1.74911 & 3.45505 & -0.42810 \\ \mathrm{H} & 0.14502 & -1.45930 & -0.27248 \\ \mathrm{H} & 2.25509 & -2.24068 & -0.80899 \\ \mathrm{H} & 4.69086 & -2.07512 & -0.72658 \\ \mathrm{H} & 4.36199 & 1.80545 & 1.08694 \\ \mathrm{H} & 1.90719 & 1.63517 & 1.02083\end{array}$




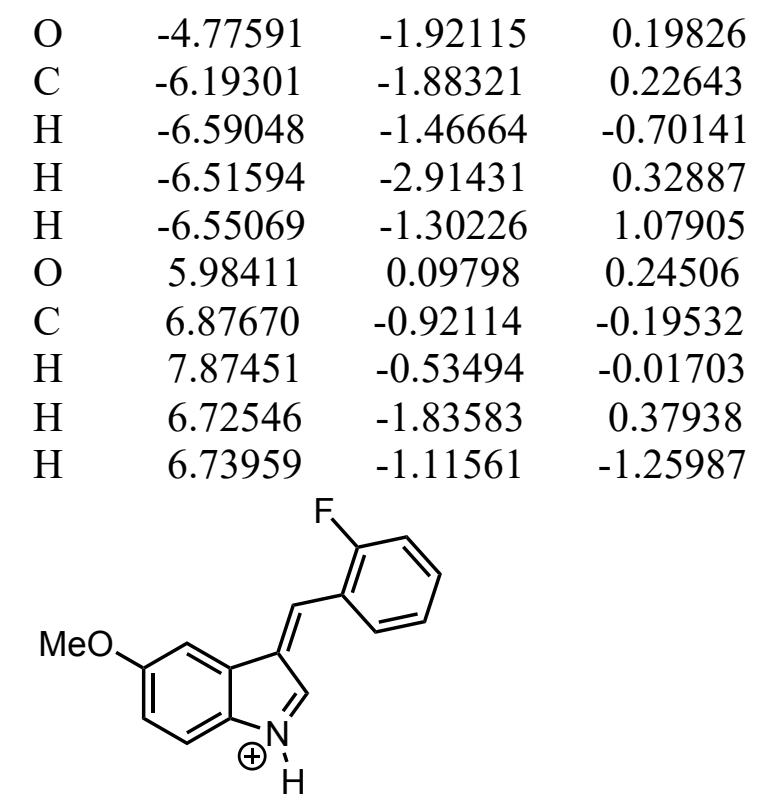

Indolium 5

$\begin{array}{lccc}\text { M06-2X/def2-TZVP Geometry } & \\ \mathrm{C} & -3.26411 & 1.69023 & -0.18244 \\ \mathrm{C} & -1.89574 & 1.51508 & -0.17706 \\ \mathrm{C} & -1.28708 & 0.26288 & -0.05018 \\ \mathrm{C} & -2.07061 & -0.86379 & 0.08084 \\ \mathrm{C} & -3.46175 & -0.71285 & 0.07365 \\ \mathrm{C} & -4.04935 & 0.55565 & -0.05652 \\ \mathrm{C} & 0.16614 & 0.48536 & -0.07870 \\ \mathrm{C} & 0.32032 & 1.90477 & -0.22731 \\ \mathrm{~N} & -0.86373 & 2.46184 & -0.28707 \\ \mathrm{C} & 1.09144 & -0.51860 & -0.06058 \\ \mathrm{C} & 2.52672 & -0.58308 & -0.04540 \\ \mathrm{C} & 3.40799 & 0.46230 & 0.26033 \\ \mathrm{C} & 4.77368 & 0.30800 & 0.27797 \\ \mathrm{C} & 5.30954 & -0.93505 & -0.03054 \\ \mathrm{C} & 4.47862 & -2.01036 & -0.33083 \\ \mathrm{C} & 3.11234 & -1.83614 & -0.32172 \\ \mathrm{H} & -3.71828 & 2.66730 & -0.27980 \\ \mathrm{H} & -1.66431 & -1.86008 & 0.19431 \\ \mathrm{H} & -5.12348 & 0.66048 & -0.05794 \\ \mathrm{H} & 1.21703 & 2.49163 & -0.31667 \\ \mathrm{H} & -1.01296 & 3.45525 & -0.40642 \\ \mathrm{H} & 0.64178 & -1.50864 & -0.07727 \\ \mathrm{~F} & 2.90954 & 1.65901 & 0.58529 \\ \mathrm{H} & 5.39652 & 1.15339 & 0.53538 \\ \mathrm{H} & 4.90112 & -2.97851 & -0.55873 \\ \mathrm{H} & 2.45964 & -2.67197 & -0.54068 \\ \mathrm{O} & -4.16080 & -1.84755 & 0.20072\end{array}$




$\begin{array}{lrrr}\text { C } & -5.57868 & -1.78442 & 0.20757 \\ \mathrm{H} & -5.92053 & -2.80812 & 0.32064 \\ \mathrm{H} & -5.93669 & -1.18425 & 1.04637 \\ \mathrm{H} & -5.95373 & -1.37506 & -0.73251 \\ \mathrm{H} & 6.38364 & -1.06489 & -0.02502\end{array}$<smiles></smiles>

\section{Indolium 6}

$\begin{array}{lrrr}\text { M06-2X/def2-TZVP Geometry } & \\ \mathrm{C} & 3.45149 & -1.78497 & -0.09467 \\ \mathrm{C} & 2.08821 & -1.55320 & -0.14035 \\ \mathrm{C} & 1.50456 & -0.29068 & -0.06955 \\ \mathrm{C} & 2.32721 & 0.83199 & 0.06188 \\ \mathrm{C} & 3.70348 & 0.62883 & 0.10915 \\ \mathrm{C} & 4.24397 & -0.65650 & 0.03065 \\ \mathrm{C} & 0.06122 & -0.46989 & -0.11516 \\ \mathrm{C} & -0.13382 & -1.87744 & -0.24800 \\ \mathrm{~N} & 1.03537 & -2.47850 & -0.26362 \\ \mathrm{C} & -0.91919 & 0.48914 & -0.13397 \\ \mathrm{C} & -2.33673 & 0.29670 & -0.01317 \\ \mathrm{C} & -3.18246 & 1.25050 & -0.60381 \\ \mathrm{C} & -4.55340 & 1.08731 & -0.56620 \\ \mathrm{C} & -5.10081 & -0.00308 & 0.10048 \\ \mathrm{C} & -4.27808 & -0.92612 & 0.74068 \\ \mathrm{C} & -2.90651 & -0.78108 & 0.68706 \\ \mathrm{H} & 3.87511 & -2.77764 & -0.14881 \\ \mathrm{O} & 1.71317 & 2.02405 & 0.12690 \\ \mathrm{H} & 5.31889 & -0.76782 & 0.07107 \\ \mathrm{H} & -1.05202 & -2.42232 & -0.40140 \\ \mathrm{H} & 1.16638 & -3.47235 & -0.39531 \\ \mathrm{H} & -0.57835 & 1.51057 & -0.27160 \\ \mathrm{H} & -5.19869 & 1.81303 & -1.04179 \\ \mathrm{H} & -4.71458 & -1.74195 & 1.30064 \\ \mathrm{H} & -2.27242 & -1.45224 & 1.25169 \\ \mathrm{H} & -6.17556 & -0.12013 & 0.14754 \\ \mathrm{H} & -2.74792 & 2.10152 & -1.11353 \\ \mathrm{H} & 4.37442 & 1.46953 & 0.20430 \\ \mathrm{C} & 2.51991 & 3.18501 & 0.29243 \\ \mathrm{H} & 1.83208 & 4.02293 & 0.34250 \\ \mathrm{H} & 3.09384 & 3.12431 & 1.21828 \\ \mathrm{H} & 3.19148 & 3.31053 & -0.55814\end{array}$


<smiles>Brc1ccc(/C=C2\C[NH2+]c3ccccc32)cc1</smiles>

\section{Indolium 7}

$\begin{array}{lccc}\text { M06-2X/def2-TZVP Geometry } & \\ \mathrm{C} & 5.47759 & -0.97911 & -0.10093 \\ \mathrm{C} & 4.09817 & -0.88486 & -0.14861 \\ \mathrm{C} & 3.39662 & 0.31002 & 0.02776 \\ \mathrm{C} & 4.10434 & 1.47983 & 0.27493 \\ \mathrm{C} & 5.48739 & 1.40953 & 0.31937 \\ \mathrm{C} & 6.16430 & 0.20017 & 0.13555 \\ \mathrm{C} & 1.97500 & -0.01182 & -0.07290 \\ \mathrm{C} & 1.92139 & -1.41252 & -0.33510 \\ \mathrm{~N} & 3.14493 & -1.89306 & -0.37774 \\ \mathrm{C} & 0.92031 & 0.86289 & -0.04130 \\ \mathrm{C} & -0.48009 & 0.58026 & 0.02014 \\ \mathrm{C} & -1.37419 & 1.55958 & -0.44850 \\ \mathrm{C} & -2.73232 & 1.32727 & -0.46804 \\ \mathrm{C} & -3.22071 & 0.12035 & 0.02594 \\ \mathrm{C} & -2.36069 & -0.84445 & 0.55270 \\ \mathrm{C} & -1.00362 & -0.61450 & 0.54858 \\ \mathrm{H} & 5.99537 & -1.91885 & -0.23811 \\ \mathrm{H} & 7.24442 & 0.18418 & 0.18202 \\ \mathrm{H} & 1.06691 & -2.03113 & -0.55980 \\ \mathrm{H} & 3.37308 & -2.85399 & -0.59521 \\ \mathrm{H} & 1.18250 & 1.91755 & -0.08590 \\ \mathrm{H} & -3.41609 & 2.07249 & -0.84901 \\ \mathrm{H} & -2.76723 & -1.74996 & 0.98061 \\ \mathrm{H} & -0.34706 & -1.32881 & 1.02750 \\ \mathrm{Br} & -5.06452 & -0.19677 & 0.02671 \\ \mathrm{H} & -0.98690 & 2.49828 & -0.82530 \\ \mathrm{H} & 6.05880 & 2.30894 & 0.50368 \\ \mathrm{H} & 3.59875 & 2.42371 & 0.43124\end{array}$


<smiles>COc1cccc(/C=C2\CNc3ccccc32)c1</smiles>

Indolium 8

$\begin{array}{lccc}\text { M06-2X/def2-TZVP Geometry } & \\ \mathrm{C} & 4.44894 & -1.20043 & -0.17988 \\ \mathrm{C} & 3.09286 & -0.94066 & -0.25952 \\ \mathrm{C} & 2.51731 & 0.28600 & 0.07833 \\ \mathrm{C} & 3.33422 & 1.31445 & 0.53052 \\ \mathrm{C} & 4.69741 & 1.07842 & 0.61177 \\ \mathrm{C} & 5.24713 & -0.15848 & 0.26338 \\ \mathrm{C} & 1.07569 & 0.14924 & -0.12084 \\ \mathrm{C} & 0.88398 & -1.18149 & -0.60188 \\ \mathrm{~N} & 2.04784 & -1.78627 & -0.67704 \\ \mathrm{C} & 0.11743 & 1.11581 & 0.01467 \\ \mathrm{C} & -1.31051 & 0.97443 & -0.03690 \\ \mathrm{C} & -1.95864 & -0.22227 & 0.28491 \\ \mathrm{C} & -3.34099 & -0.32166 & 0.18277 \\ \mathrm{C} & -4.07867 & 0.78756 & -0.24272 \\ \mathrm{C} & -3.43496 & 1.98413 & -0.53738 \\ \mathrm{C} & -2.06519 & 2.09723 & -0.41900 \\ \mathrm{H} & 4.86762 & -2.16173 & -0.44563 \\ \mathrm{H} & 6.31514 & -0.30652 & 0.34416 \\ \mathrm{H} & -0.02263 & -1.65537 & -0.94385 \\ \mathrm{H} & 2.18100 & -2.72397 & -1.03181 \\ \mathrm{H} & 0.48224 & 2.12977 & 0.16127 \\ \mathrm{O} & -3.87393 & -1.50379 & 0.52802 \\ \mathrm{H} & -4.02266 & 2.83667 & -0.84937 \\ \mathrm{H} & -1.56737 & 3.03140 & -0.64293 \\ \mathrm{H} & -1.42010 & -1.06424 & 0.69840 \\ \mathrm{H} & 5.35277 & 1.86699 & 0.95544 \\ \mathrm{H} & 2.92658 & 2.27557 & 0.81510 \\ \mathrm{H} & -5.15505 & 0.73345 & -0.31910 \\ \mathrm{C} & -5.28589 & -1.64827 & 0.48615 \\ \mathrm{H} & -5.49101 & -2.66288 & 0.81202 \\ \mathrm{H} & -5.76864 & -0.94175 & 1.16426 \\ \mathrm{H} & -5.66193 & -1.50743 & -0.52916\end{array}$


<smiles>Fc1ccccc1/C=C1\CNc2ccccc21</smiles>

\section{Indolium 9}

$\begin{array}{lccc}\text { M06-2X/def2-TZVP Geometry } & \\ \mathrm{C} & -4.16214 & 0.67398 & -0.10656 \\ \mathrm{C} & -2.78171 & 0.74867 & -0.12679 \\ \mathrm{C} & -1.94022 & -0.35334 & 0.02296 \\ \mathrm{C} & -2.50397 & -1.60880 & 0.20820 \\ \mathrm{C} & -3.88651 & -1.71026 & 0.22860 \\ \mathrm{C} & -4.70429 & -0.58817 & 0.07409 \\ \mathrm{C} & -0.55997 & 0.14111 & -0.04791 \\ \mathrm{C} & -0.68295 & 1.55422 & -0.24470 \\ \mathrm{~N} & -1.95388 & 1.87415 & -0.29235 \\ \mathrm{C} & 0.54169 & -0.67027 & -0.01281 \\ \mathrm{C} & 1.96203 & -0.47342 & -0.02469 \\ \mathrm{C} & 2.64472 & 0.73338 & 0.18418 \\ \mathrm{C} & 4.01553 & 0.82697 & 0.18232 \\ \mathrm{C} & 4.76262 & -0.32080 & -0.04696 \\ \mathrm{C} & 4.13627 & -1.54712 & -0.25146 \\ \mathrm{C} & 2.76173 & -1.61944 & -0.22541 \\ \mathrm{H} & -4.78759 & 1.54862 & -0.22439 \\ \mathrm{H} & -5.77879 & -0.70595 & 0.09776 \\ \mathrm{H} & 0.08227 & 2.29886 & -0.37386 \\ \mathrm{H} & -2.29434 & 2.81567 & -0.43768 \\ \mathrm{H} & 0.28265 & -1.72594 & 0.01935 \\ \mathrm{H} & 4.72437 & -2.43803 & -0.41916 \\ \mathrm{H} & 2.26798 & -2.57212 & -0.37070 \\ \mathrm{~F} & 1.94214 & 1.84230 & 0.42899 \\ \mathrm{H} & -4.34461 & -2.67956 & 0.37021 \\ \mathrm{H} & -1.89274 & -2.49230 & 0.33892 \\ \mathrm{H} & 5.84263 & -0.25610 & -0.05606 \\ \mathrm{H} & 4.47919 & 1.78684 & 0.36270\end{array}$


<smiles></smiles>

\section{Indolium 10}

$\begin{array}{lccc}\text { M06-2X/def2-TZVP Geometry } & \\ \mathrm{C} & 2.35497 & 1.03607 & -0.19183 \\ \mathrm{C} & 0.97530 & 0.94719 & -0.20877 \\ \mathrm{C} & 0.26640 & -0.24041 & -0.02091 \\ \mathrm{C} & 0.97411 & -1.41457 & 0.20759 \\ \mathrm{C} & 2.35669 & -1.36174 & 0.22337 \\ \mathrm{C} & 3.03268 & -0.15245 & 0.02690 \\ \mathrm{C} & -1.15277 & 0.09516 & -0.08951 \\ \mathrm{C} & -1.19819 & 1.49715 & -0.34685 \\ \mathrm{~N} & 0.02860 & 1.96663 & -0.41486 \\ \mathrm{C} & -2.22033 & -0.76210 & -0.03907 \\ \mathrm{C} & -3.61503 & -0.44840 & 0.05086 \\ \mathrm{C} & -4.53449 & -1.39493 & -0.43396 \\ \mathrm{C} & -5.88785 & -1.12156 & -0.42462 \\ \mathrm{C} & -6.34501 & 0.07794 & 0.10963 \\ \mathrm{C} & -5.45092 & 1.00191 & 0.64650 \\ \mathrm{C} & -4.09616 & 0.74503 & 0.61994 \\ \mathrm{H} & 2.88381 & 1.96785 & -0.33662 \\ \mathrm{Br} & 4.91225 & -0.13745 & 0.06533 \\ \mathrm{H} & -2.05298 & 2.12292 & -0.55022 \\ \mathrm{H} & 0.26200 & 2.92583 & -0.63510 \\ \mathrm{H} & -1.97984 & -1.82159 & -0.09241 \\ \mathrm{H} & -5.82167 & 1.90783 & 1.10622 \\ \mathrm{H} & -3.41068 & 1.42474 & 1.10903 \\ \mathrm{H} & 2.93126 & -2.26152 & 0.39221 \\ \mathrm{H} & 0.46968 & -2.35757 & 0.37240 \\ \mathrm{H} & -7.40711 & 0.28383 & 0.13572 \\ \mathrm{H} & -6.58956 & -1.84372 & -0.81854 \\ \mathrm{H} & -4.17013 & -2.33023 & -0.84086\end{array}$


<smiles></smiles>

Indolium 11

$\begin{array}{lccc}\text { M06-2X/def2-TZVP Geometry } & \\ \mathrm{C} & 1.92011 & -1.44962 & 0.25018 \\ \mathrm{C} & 0.54993 & -1.26754 & 0.27437 \\ \mathrm{C} & -0.07194 & -0.04219 & 0.03241 \\ \mathrm{C} & 0.71158 & 1.06624 & -0.25214 \\ \mathrm{C} & 2.09313 & 0.92454 & -0.27506 \\ \mathrm{C} & 2.68663 & -0.33111 & -0.02743 \\ \mathrm{C} & -1.51229 & -0.27413 & 0.10986 \\ \mathrm{C} & -1.65612 & -1.65871 & 0.42953 \\ \mathrm{~N} & -0.46566 & -2.20750 & 0.52254 \\ \mathrm{C} & -2.51670 & 0.65010 & 0.01092 \\ \mathrm{C} & -3.93039 & 0.42559 & -0.07161 \\ \mathrm{C} & -4.78690 & 1.44872 & 0.36949 \\ \mathrm{C} & -6.15542 & 1.26422 & 0.36353 \\ \mathrm{C} & -6.68899 & 0.07673 & -0.12464 \\ \mathrm{C} & -5.85575 & -0.92442 & -0.61847 \\ \mathrm{C} & -4.48678 & -0.75568 & -0.59432 \\ \mathrm{H} & 2.38484 & -2.41030 & 0.42452 \\ \mathrm{Br} & 4.55350 & -0.50046 & -0.11950 \\ \mathrm{H} & -2.55265 & -2.21322 & 0.65775 \\ \mathrm{H} & -0.30002 & -3.17011 & 0.78513 \\ \mathrm{H} & -2.20522 & 1.69227 & 0.01174 \\ \mathrm{H} & -6.28334 & -1.82222 & -1.04350 \\ \mathrm{H} & -3.84560 & -1.49821 & -1.05113 \\ \mathrm{O} & 2.85455 & 1.99128 & -0.59913 \\ \mathrm{H} & 0.29340 & 2.03945 & -0.47475 \\ \mathrm{H} & -7.76223 & -0.05998 & -0.14843 \\ \mathrm{H} & -6.80930 & 2.04630 & 0.72398 \\ \mathrm{H} & -4.36338 & 2.37417 & 0.73998 \\ \mathrm{C} & 3.36600 & 2.72906 & 0.51484 \\ \mathrm{H} & 2.54325 & 3.16986 & 1.08187 \\ \mathrm{H} & 3.96300 & 2.08670 & 1.16391 \\ \mathrm{H} & 3.99115 & 3.51270 & 0.09796\end{array}$


<smiles></smiles>

Indolium 12

$\begin{array}{lccc}\text { M06-2X/def2-TZVP Geometry } & \\ \mathrm{C} & -0.37281 & 3.09111 & -0.25409 \\ \mathrm{C} & 0.84456 & 2.43538 & -0.23477 \\ \mathrm{C} & 0.97493 & 1.04900 & -0.12752 \\ \mathrm{C} & -0.16863 & 0.26951 & -0.02593 \\ \mathrm{C} & -1.41517 & 0.89060 & -0.04912 \\ \mathrm{C} & -1.49810 & 2.28804 & -0.16090 \\ \mathrm{C} & 2.40741 & 0.75587 & -0.12058 \\ \mathrm{C} & 3.05555 & 2.02285 & -0.25321 \\ \mathrm{~N} & 2.14539 & 2.96737 & -0.32022 \\ \mathrm{C} & 3.01104 & -0.46971 & -0.10656 \\ \mathrm{C} & 4.40666 & -0.77577 & 0.04502 \\ \mathrm{C} & 4.88310 & -1.97503 & -0.50887 \\ \mathrm{C} & 6.22583 & -2.29188 & -0.43898 \\ \mathrm{C} & 7.10261 & -1.43957 & 0.22232 \\ \mathrm{C} & 6.63584 & -0.27453 & 0.82519 \\ \mathrm{C} & 5.29895 & 0.05827 & 0.74017 \\ \mathrm{H} & -0.44560 & 4.16752 & -0.33505 \\ \mathrm{H} & 4.10535 & 2.24039 & -0.37190 \\ \mathrm{H} & 2.35051 & 3.94737 & -0.46277 \\ \mathrm{H} & 2.35258 & -1.32420 & -0.24576 \\ \mathrm{H} & 7.31618 & 0.35641 & 1.38077 \\ \mathrm{H} & 4.92463 & 0.92105 & 1.27558 \\ \mathrm{H} & -0.11554 & -0.80840 & 0.07129 \\ \mathrm{H} & 8.15118 & -1.69719 & 0.29402 \\ \mathrm{H} & 6.59037 & -3.20671 & -0.88553 \\ \mathrm{H} & 4.19229 & -2.63805 & -1.01509 \\ \mathrm{H} & -2.47964 & 2.74446 & -0.17130 \\ \mathrm{C} & -3.74479 & -2.17392 & 0.21282 \\ \mathrm{O} & -2.59128 & -1.32099 & 0.15536 \\ \mathrm{~B} & -2.73125 & 0.02548 & 0.05447 \\ \mathrm{O} & -3.91946 & 0.67286 & 0.03078 \\ \mathrm{C} & -5.15501 & -0.06358 & 0.15670 \\ \mathrm{C} & -4.93961 & -1.46828 & -0.40772 \\ \mathrm{C} & -3.93818 & -2.37717 & 1.26956 \\ \mathrm{C} & -3.40403 & -3.46950 & -0.48984 \\ & -5.53432 & -0.08147 & 1.63142 \\ \mathrm{H} & -6.19113 & 0.68951 & -0.65740\end{array}$




\begin{tabular}{lrrr}
$\mathrm{H}$ & -4.77671 & -1.38902 & -1.48762 \\
$\mathrm{H}$ & -5.83855 & -2.06782 & -0.25367 \\
$\mathrm{H}$ & -7.15847 & 0.19010 & -0.59393 \\
$\mathrm{H}$ & -6.29818 & 1.70561 & -0.27781 \\
$\mathrm{H}$ & -5.89059 & 0.73829 & -1.70410 \\
$\mathrm{H}$ & -6.47127 & -0.62053 & 1.77653 \\
$\mathrm{H}$ & -4.76173 & -0.55788 & 2.23602 \\
$\mathrm{H}$ & -5.66232 & 0.93960 & 1.99008 \\
$\mathrm{H}$ & -3.18734 & -3.28091 & -1.54219 \\
$\mathrm{H}$ & -2.53137 & -3.93214 & -0.03040 \\
$\mathrm{H}$ & -4.24026 & -4.16605 & -0.42551 \\
& & & \\
\hline
\end{tabular}

\section{Indolium 13}

$\begin{array}{lccc}\text { M06-2X/def2-TZVP Geometry } & \\ \mathrm{C} & 1.64277 & 1.82466 & -0.00000 \\ \mathrm{C} & 0.30572 & 1.48459 & -0.00000 \\ \mathrm{C} & -0.14280 & 0.15827 & -0.00000 \\ \mathrm{C} & 0.77162 & -0.87260 & -0.00000 \\ \mathrm{C} & 2.13453 & -0.55180 & -0.00000 \\ \mathrm{C} & 2.56059 & 0.78667 & -0.00000 \\ \mathrm{C} & -1.60619 & 0.20953 & -0.00000 \\ \mathrm{C} & -1.93872 & 1.60485 & -0.00000 \\ \mathrm{~N} & -0.83472 & 2.30829 & -0.00000 \\ \mathrm{C} & -2.49194 & -0.82425 & -0.00000 \\ \mathrm{C} & -3.92080 & -0.72568 & -0.00000 \\ \mathrm{C} & -4.67858 & -1.82872 & -0.00000 \\ \mathrm{H} & 1.97487 & 2.85430 & -0.00000 \\ \mathrm{H} & -2.90807 & 2.07843 & -0.00000 \\ \mathrm{H} & -0.80508 & 3.31990 & -0.00000 \\ \mathrm{H} & -2.08125 & -1.83062 & -0.00000 \\ \mathrm{H} & -4.23172 & -2.81605 & -0.00000 \\ \mathrm{H} & 0.48709 & -1.91623 & -0.00000 \\ \mathrm{H} & 3.61399 & 1.02161 & -0.00000 \\ \mathrm{O} & 2.96751 & -1.59740 & -0.00000 \\ \mathrm{C} & 4.36829 & -1.36391 & 0.00000 \\ \mathrm{H} & 4.83173 & -2.34506 & 0.00000 \\ \mathrm{H} & 4.66994 & -0.81627 & -0.89489 \\ \mathrm{H} & 4.66994 & -0.81627 & 0.89489 \\ \mathrm{H} & -4.38649 & 0.25219 & -0.00000 \\ \mathrm{H} & -5.75896 & -1.77439 & -0.00000\end{array}$


<smiles>O=C1Nc2ccccc2/C1=C1\C=[NH+]c2ccc(Br)cc21</smiles>

\section{Indolium 14}

$\begin{array}{lccc}\text { M06-2X/def2-TZVP Geometry } & \\ \mathrm{C} & -4.14546 & -2.15936 & -0.10197 \\ \mathrm{C} & -3.56351 & -0.91189 & -0.18550 \\ \mathrm{C} & -2.20277 & -0.69673 & 0.14889 \\ \mathrm{C} & -1.44607 & -1.76207 & 0.66269 \\ \mathrm{C} & -2.02522 & -3.00715 & 0.76233 \\ \mathrm{C} & -3.35330 & -3.20137 & 0.36370 \\ \mathrm{C} & -1.93312 & 0.68823 & -0.02054 \\ \mathrm{C} & -3.27480 & 1.32971 & -0.43509 \\ \mathrm{~N} & -4.15628 & 0.28609 & -0.54727 \\ \mathrm{O} & -3.49602 & 2.48666 & -0.65260 \\ \mathrm{C} & -0.79118 & 1.44426 & 0.07536 \\ \mathrm{C} & 0.63008 & 1.09829 & 0.13330 \\ \mathrm{C} & 1.32403 & 2.29557 & 0.34714 \\ \mathrm{~N} & 0.37366 & 3.32838 & 0.40728 \\ \mathrm{C} & -0.84033 & 2.86891 & 0.22042 \\ \mathrm{C} & 1.36172 & -0.06062 & -0.09675 \\ \mathrm{C} & 2.74634 & 0.01411 & -0.03114 \\ \mathrm{C} & 3.41420 & 1.20886 & 0.23816 \\ \mathrm{C} & 2.70013 & 2.38281 & 0.41493 \\ \mathrm{Br} & 3.75295 & -1.54859 & -0.32959 \\ \mathrm{H} & -5.18016 & -2.31997 & -0.37109 \\ \mathrm{H} & -0.43902 & -1.60276 & 1.01756 \\ \mathrm{H} & -1.45954 & -3.83829 & 1.15817 \\ \mathrm{H} & -3.78607 & -4.19048 & 0.44195 \\ \mathrm{H} & -5.12417 & 0.41719 & -0.79749 \\ \mathrm{H} & 0.59515 & 4.30133 & 0.57247 \\ \mathrm{H} & -1.71143 & 3.50415 & 0.23117 \\ \mathrm{H} & 0.89204 & -0.99544 & -0.35979 \\ \mathrm{H} & 4.49385 & 1.21814 & 0.28415 \\ \mathrm{H} & 3.20812 & 3.32292 & 0.58350\end{array}$


<smiles>COc1ccc2c(c1)/C(=C1/C(=O)Nc3ccccc31)C=[NH+]2</smiles>

Indolium 15

$\begin{array}{lccc}\text { M06-2X/def2-TZVP Geometry } & \\ \mathrm{C} & -4.13464 & 1.61538 & 0.12992 \\ \mathrm{C} & -3.31863 & 0.50553 & 0.18420 \\ \mathrm{C} & -1.93590 & 0.57673 & -0.11028 \\ \mathrm{C} & -1.39295 & 1.79088 & -0.55203 \\ \mathrm{C} & -2.20547 & 2.90282 & -0.62268 \\ \mathrm{C} & -3.55420 & 2.81477 & -0.26542 \\ \mathrm{C} & -1.40203 & -0.74077 & 0.01060 \\ \mathrm{C} & -2.60229 & -1.64854 & 0.34913 \\ \mathrm{~N} & -3.67225 & -0.80304 & 0.47574 \\ \mathrm{O} & -2.60260 & -2.83690 & 0.50905 \\ \mathrm{C} & -0.13559 & -1.25368 & -0.07546 \\ \mathrm{C} & 1.19604 & -0.63746 & -0.08341 \\ \mathrm{C} & 2.11232 & -1.67605 & -0.31075 \\ \mathrm{~N} & 1.37807 & -2.86930 & -0.42270 \\ \mathrm{C} & 0.09713 & -2.66109 & -0.25905 \\ \mathrm{C} & 1.68286 & 0.62543 & 0.19301 \\ \mathrm{C} & 3.06766 & 0.83842 & 0.17422 \\ \mathrm{C} & 3.95451 & -0.20869 & -0.10343 \\ \mathrm{C} & 3.47636 & -1.49091 & -0.33288 \\ \mathrm{H} & -5.18745 & 1.55477 & 0.36839 \\ \mathrm{H} & -0.36482 & 1.85054 & -0.87477 \\ \mathrm{H} & -1.80340 & 3.84608 & -0.96345 \\ \mathrm{H} & -4.17260 & 3.70148 & -0.31905 \\ \mathrm{H} & -4.60134 & -1.13274 & 0.68620 \\ \mathrm{H} & 1.78624 & -3.77609 & -0.60756 \\ \mathrm{H} & -0.63453 & -3.45097 & -0.31116 \\ \mathrm{H} & 1.05236 & 1.45781 & 0.46447 \\ \mathrm{H} & 5.01971 & -0.03574 & -0.11836 \\ \mathrm{H} & 4.16071 & -2.31042 & -0.50821 \\ \mathrm{O} & 3.45149 & 2.09331 & 0.44360 \\ \mathrm{C} & 4.83850 & 2.39423 & 0.46721 \\ \mathrm{H} & 4.90950 & 3.44914 & 0.71155 \\ \mathrm{H} & 5.34999 & 1.80599 & 1.23165 \\ \mathrm{H} & 5.29169 & 2.21334 & -0.50949\end{array}$


<smiles>O=C1Nc2ccccc2/C1=C1\C=[NH+]c2ccc(Cl)cc21</smiles>

Indolium 16

$\begin{array}{lccc}\text { M06-2X/def2-TZVP Geometry } & \\ \mathrm{C} & 4.10675 & 1.49497 & -0.10859 \\ \mathrm{C} & 3.26432 & 0.40499 & -0.17296 \\ \mathrm{C} & 1.88265 & 0.50780 & 0.12696 \\ \mathrm{C} & 1.36993 & 1.73160 & 0.58613 \\ \mathrm{C} & 2.20865 & 2.82054 & 0.66632 \\ \mathrm{C} & 3.55547 & 2.70207 & 0.30229 \\ \mathrm{C} & 1.31638 & -0.78869 & -0.00903 \\ \mathrm{C} & 2.49147 & -1.72473 & -0.36544 \\ \mathrm{~N} & 3.58463 & -0.90628 & -0.48162 \\ \mathrm{O} & 2.45563 & -2.90873 & -0.54334 \\ \mathrm{C} & 0.03401 & -1.27122 & 0.07641 \\ \mathrm{C} & -1.27744 & -0.62062 & 0.08324 \\ \mathrm{C} & -2.22192 & -1.62928 & 0.31035 \\ \mathrm{~N} & -1.52315 & -2.84245 & 0.42790 \\ \mathrm{C} & -0.23428 & -2.66656 & 0.26388 \\ \mathrm{C} & -1.73153 & 0.66168 & -0.19904 \\ \mathrm{C} & -3.10024 & 0.89332 & -0.17242 \\ \mathrm{C} & -4.02002 & -0.11743 & 0.10878 \\ \mathrm{C} & -3.58507 & -1.41160 & 0.33924 \\ \mathrm{H} & 5.15711 & 1.41300 & -0.35120 \\ \mathrm{H} & 0.34490 & 1.81154 & 0.91522 \\ \mathrm{H} & 1.83295 & 3.76972 & 1.02043 \\ \mathrm{H} & 4.19510 & 3.57314 & 0.36445 \\ \mathrm{H} & 4.50450 & -1.25652 & -0.70015 \\ \mathrm{H} & -1.95657 & -3.73697 & 0.61490 \\ \mathrm{H} & 0.47571 & -3.47610 & 0.31847 \\ \mathrm{H} & -1.06710 & 1.46619 & -0.47315 \\ \mathrm{H} & -5.07516 & 0.11637 & 0.12079 \\ \mathrm{H} & -4.29024 & -2.21201 & 0.51881 \\ \mathrm{Cl} & -3.67949 & 2.47774 & -0.50839\end{array}$


<smiles></smiles>

\section{Indolium 17}

$\begin{array}{lccc}\text { M06-2X/def2-TZVP Geometry } & \\ \mathrm{C} & 4.26827 & -1.02896 & 0.12926 \\ \mathrm{C} & 3.24808 & -0.10161 & 0.15357 \\ \mathrm{C} & 1.89864 & -0.46745 & -0.07038 \\ \mathrm{C} & 1.59548 & -1.79326 & -0.41059 \\ \mathrm{C} & 2.61171 & -2.72361 & -0.45048 \\ \mathrm{C} & 3.92704 & -2.34394 & -0.16344 \\ \mathrm{C} & 1.11405 & 0.71962 & -0.00442 \\ \mathrm{C} & 2.11457 & 1.87061 & 0.22571 \\ \mathrm{~N} & 3.33906 & 1.26759 & 0.34778 \\ \mathrm{O} & 1.87789 & 3.04205 & 0.31944 \\ \mathrm{C} & -0.23503 & 0.95560 & -0.06620 \\ \mathrm{C} & -1.40971 & 0.08848 & 0.02030 \\ \mathrm{C} & -2.52270 & 0.89509 & -0.23652 \\ \mathrm{~N} & -2.05251 & 2.20282 & -0.46417 \\ \mathrm{C} & -0.75080 & 2.26790 & -0.33809 \\ \mathrm{C} & -1.64611 & -1.22802 & 0.40929 \\ \mathrm{C} & -2.95149 & -1.68732 & 0.45425 \\ \mathrm{C} & -4.05057 & -0.87759 & 0.13647 \\ \mathrm{C} & -3.82977 & 0.45443 & -0.19962 \\ \mathrm{H} & 5.29507 & -0.74492 & 0.31331 \\ \mathrm{H} & 0.58915 & -2.07560 & -0.68044 \\ \mathrm{H} & 2.39764 & -3.74944 & -0.71393 \\ \mathrm{H} & 4.70898 & -3.09183 & -0.19238 \\ \mathrm{H} & 4.18780 & 1.78983 & 0.50002 \\ \mathrm{H} & -2.64283 & 2.99096 & -0.69486 \\ \mathrm{H} & -0.19574 & 3.18210 & -0.47455 \\ \mathrm{H} & -0.84229 & -1.88117 & 0.71356 \\ \mathrm{C} & -5.43947 & -1.44370 & 0.17589 \\ \mathrm{H} & -4.65809 & 1.12052 & -0.40629 \\ \mathrm{H} & -3.13572 & -2.71050 & 0.75750 \\ \mathrm{H} & -6.19219 & -0.66097 & 0.10799 \\ \mathrm{H} & -5.59060 & -2.13248 & -0.65731 \\ \mathrm{H} & -5.60327 & -2.00325 & 1.09672\end{array}$


<smiles>O=C1Nc2ccccc2/C1=C1\C=[NH+]c2ccc(F)cc21</smiles>

\section{Indolium 18}

$\begin{array}{lccc}\text { M06-2X/def2-TZVP Geometry } & \\ \mathrm{C} & -4.10327 & -1.00617 & -0.12101 \\ \mathrm{C} & -3.09609 & -0.06495 & -0.16174 \\ \mathrm{C} & -1.74562 & -0.40169 & 0.10662 \\ \mathrm{C} & -1.43217 & -1.70908 & 0.51066 \\ \mathrm{C} & -2.43523 & -2.65063 & 0.56745 \\ \mathrm{C} & -3.74969 & -2.30179 & 0.23444 \\ \mathrm{C} & -0.97781 & 0.79022 & 0.00633 \\ \mathrm{C} & -1.99018 & 1.91778 & -0.29037 \\ \mathrm{~N} & -3.20325 & 1.29219 & -0.41527 \\ \mathrm{O} & -1.76489 & 3.08658 & -0.42395 \\ \mathrm{C} & 0.36713 & 1.05368 & 0.08021 \\ \mathrm{C} & 1.55338 & 0.19739 & 0.03293 \\ \mathrm{C} & 2.65539 & 1.02921 & 0.26995 \\ \mathrm{~N} & 2.16675 & 2.33554 & 0.44595 \\ \mathrm{C} & 0.86461 & 2.37919 & 0.30819 \\ \mathrm{C} & 1.78523 & -1.13167 & -0.30103 \\ \mathrm{C} & 3.09931 & -1.56383 & -0.31483 \\ \mathrm{C} & 4.18395 & -0.74233 & -0.03005 \\ \mathrm{C} & 3.96686 & 0.59463 & 0.25544 \\ \mathrm{H} & -5.12961 & -0.74612 & -0.33970 \\ \mathrm{H} & -0.42955 & -1.96581 & 0.81748 \\ \mathrm{H} & -2.21385 & -3.66111 & 0.87951 \\ \mathrm{H} & -4.52138 & -3.05969 & 0.27758 \\ \mathrm{H} & -4.05711 & 1.79523 & -0.60094 \\ \mathrm{H} & 2.74452 & 3.13964 & 0.65232 \\ \mathrm{H} & 0.29816 & 3.29110 & 0.40771 \\ \mathrm{H} & 1.00686 & -1.82273 & -0.58511 \\ \mathrm{H} & 4.79422 & 1.26552 & 0.44360 \\ \mathrm{~F} & 3.33632 & -2.83366 & -0.62601 \\ \mathrm{H} & 5.18069 & -1.15953 & -0.06061\end{array}$




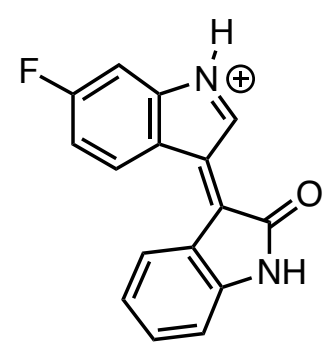

Indolium 19

M06-2X/def2-TZVP Geometry

$\begin{array}{lccc}\mathrm{C} & -4.25804 & -0.97696 & -0.12972 \\ \mathrm{C} & -3.22492 & -0.06355 & -0.15484 \\ \mathrm{C} & -1.88018 & -0.44851 & 0.07356 \\ \mathrm{C} & -1.59673 & -1.77877 & 0.42021 \\ \mathrm{C} & -2.62546 & -2.69321 & 0.46127 \\ \mathrm{C} & -3.93546 & -2.29479 & 0.16884 \\ \mathrm{C} & -1.07991 & 0.72412 & 0.00519 \\ \mathrm{C} & -2.06301 & 1.89000 & -0.23217 \\ \mathrm{~N} & -3.29655 & 1.30467 & -0.35479 \\ \mathrm{O} & -1.80666 & 3.05617 & -0.32992 \\ \mathrm{C} & 0.27418 & 0.94316 & 0.06947 \\ \mathrm{C} & 1.43576 & 0.05987 & -0.02153 \\ \mathrm{C} & 2.55989 & 0.85257 & 0.24213 \\ \mathrm{~N} & 2.10856 & 2.16243 & 0.47823 \\ \mathrm{C} & 0.80517 & 2.24320 & 0.35045 \\ \mathrm{C} & 1.64426 & -1.25927 & -0.41931 \\ \mathrm{C} & 2.93597 & -1.75368 & -0.46929 \\ \mathrm{C} & 4.00990 & -0.93342 & -0.14124 \\ \mathrm{C} & 3.86181 & 0.39736 & 0.20615 \\ \mathrm{H} & -5.28041 & -0.67976 & -0.31747 \\ \mathrm{H} & -0.59555 & -2.07448 & 0.69539 \\ \mathrm{H} & -2.42793 & -3.72094 & 0.73010 \\ \mathrm{H} & -4.72790 & -3.03159 & 0.19886 \\ \mathrm{H} & -4.13811 & 1.83875 & -0.50698 \\ \mathrm{H} & 2.70828 & 2.94166 & 0.71491 \\ \mathrm{H} & 0.26251 & 3.16409 & 0.49250 \\ \mathrm{H} & 0.82600 & -1.89307 & -0.72546 \\ \mathrm{H} & 4.72390 & 1.01699 & 0.41059 \\ \mathrm{~F} & 5.23358 & -1.43972 & -0.19114 \\ \mathrm{H} & 3.13670 & -2.77195 & -0.77202\end{array}$




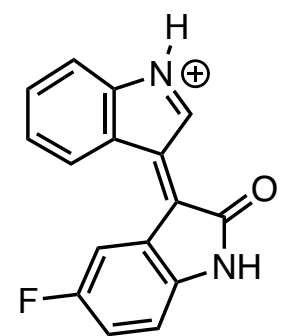

\section{Indolium 20}

M06-2X/def2-TZVP Geometry

$\begin{array}{lrrr}\mathrm{C} & 3.89802 & 0.13942 & 0.20466 \\ \mathrm{C} & 2.67002 & 0.77199 & 0.19201 \\ \mathrm{C} & 1.46587 & 0.05789 & -0.00194 \\ \mathrm{C} & 1.50925 & -1.31745 & -0.26737 \\ \mathrm{C} & 2.73867 & -1.92769 & -0.26234 \\ \mathrm{C} & 3.92003 & -1.22953 & -0.01567 \\ \mathrm{C} & 0.39856 & 1.00436 & 0.00672 \\ \mathrm{C} & 1.06694 & 2.38654 & 0.16876 \\ \mathrm{~N} & 2.40337 & 2.12835 & 0.31551 \\ \mathrm{O} & 0.53117 & 3.45811 & 0.20128 \\ \mathrm{C} & -0.96334 & 0.87931 & -0.06008 \\ \mathrm{C} & -1.87445 & -0.25895 & 0.07764 \\ \mathrm{C} & -3.15615 & 0.21752 & -0.22529 \\ \mathrm{~N} & -3.03706 & 1.58902 & -0.52409 \\ \mathrm{C} & -1.80038 & 1.99720 & -0.40192 \\ \mathrm{C} & -1.75425 & -1.56589 & 0.53869 \\ \mathrm{C} & -2.89370 & -2.35609 & 0.60828 \\ \mathrm{C} & -4.14576 & -1.86460 & 0.24464 \\ \mathrm{C} & -4.30186 & -0.54790 & -0.16581 \\ \mathrm{H} & 4.81586 & 0.68773 & 0.36425 \\ \mathrm{H} & 0.63095 & -1.89261 & -0.51617 \\ \mathrm{~F} & 2.81617 & -3.23265 & -0.51144 \\ \mathrm{H} & 4.85410 & -1.77612 & -0.02300 \\ \mathrm{H} & 3.08810 & 2.85944 & 0.42939 \\ \mathrm{H} & -3.80877 & 2.18351 & -0.79744 \\ \mathrm{H} & -1.49971 & 3.01599 & -0.58789 \\ \mathrm{H} & -0.80953 & -1.96248 & 0.87806 \\ \mathrm{H} & -5.27468 & -0.14215 & -0.40928 \\ \mathrm{H} & -2.80809 & -3.37431 & 0.96222 \\ \mathrm{H} & -5.01212 & -2.50848 & 0.30673\end{array}$




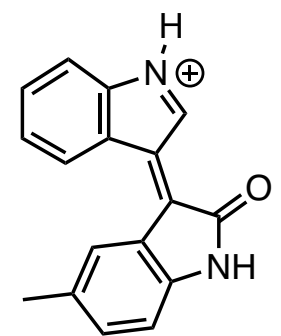

Indolium 21

M06-2X/def2-TZVP Geometry

$\begin{array}{llll}\text { C } & 3.88581 & -0.24021 & -0.23133\end{array}$

$\begin{array}{llll}\text { C } & 2.65056 & -0.85470 & -0.20394\end{array}$

$\begin{array}{llll}\text { C } & 1.46290 & -0.11376 & -0.00130\end{array}$

$\begin{array}{llll}\mathrm{C} & 1.54613 & 1.26420 & 0.26307\end{array}$

$\begin{array}{llll}\mathrm{C} & 2.77157 & 1.89696 & 0.25624\end{array}$

$\begin{array}{llll}\text { C } & 3.92005 & 1.12869 & -0.01358\end{array}$

$\begin{array}{llll}\text { C } & 0.38027 & -1.03315 & 0.00151\end{array}$

$\begin{array}{llll}\text { C } & 1.01498 & -2.43123 & -0.16380\end{array}$

$\begin{array}{llll}\mathrm{N} & 2.35751 & -2.20394 & -0.31903\end{array}$

$\begin{array}{llll}\mathrm{O} & 0.45614 & -3.49078 & -0.19222\end{array}$

$\begin{array}{llll}\text { C } & -0.98292 & -0.88271 & 0.07925\end{array}$

$\begin{array}{llll}\text { C } & -1.87032 & 0.27039 & -0.07647\end{array}$

$\begin{array}{llll}\text { C } & -3.16167 & -0.17594 & 0.23307\end{array}$

$\begin{array}{llll}\mathrm{N} & -3.06960 & -1.54140 & 0.55619\end{array}$

$\begin{array}{llll}\mathrm{C} & -1.83696 & -1.97412 & 0.44071\end{array}$

$\begin{array}{llll}\text { C } & -1.72369 & 1.56747 & -0.55942\end{array}$

$\begin{array}{llll}\text { C } & -2.84662 & 2.37775 & -0.64581\end{array}$

$\begin{array}{llll}\text { C } & -4.10961 & 1.91727 & -0.27579\end{array}$

$\begin{array}{llll}\text { C } & -4.29204 & 0.61258 & 0.15787\end{array}$

$\begin{array}{llll}\mathrm{H} & 4.79444 & -0.80181 & -0.39868\end{array}$

$\begin{array}{llll}\mathrm{H} & 0.65756 & 1.82383 & 0.51748\end{array}$

$\begin{array}{llll}\text { C } & 2.90661 & 3.36661 & 0.53679\end{array}$

$\mathrm{H} \quad 4.88125 \quad 1.63005 \quad-0.02712$

$\begin{array}{llll}\mathrm{H} & 3.02499 & -2.94920 & -0.44180\end{array}$

$\mathrm{H} \quad-3.85153 \quad-2.11665 \quad 0.83925$

$\begin{array}{llll}\mathrm{H} & -1.55709 & -2.99501 & 0.64497\end{array}$

$\begin{array}{llll}\mathrm{H} & -0.76879 & 1.93788 & -0.90039\end{array}$

$\begin{array}{llll}\mathrm{H} & -5.27301 & 0.23056 & 0.40696\end{array}$

$\begin{array}{llll}\mathrm{H} & -2.74147 & 3.38753 & -1.01832\end{array}$

$\mathrm{H} \quad-4.96289 \quad 2.57697 \quad-0.35155$

$\begin{array}{llll}\mathrm{H} & 3.57853 & 3.54104 & 1.37776\end{array}$

$\begin{array}{llll}\mathrm{H} & 3.32017 & 3.88830 & -0.32745\end{array}$

$\begin{array}{llll}\mathrm{H} & 1.94309 & 3.81354 & 0.77413\end{array}$ 


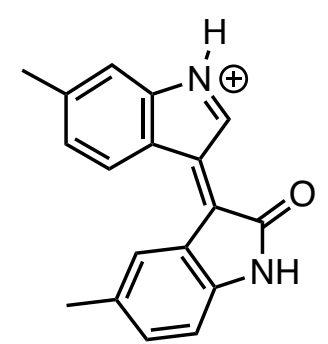

Indolium 22

M06-2X/def2-TZVP Geometry

$\begin{array}{llll}\mathrm{C} & 4.19266 & 0.33728 & -0.26109\end{array}$

$\begin{array}{llll}\text { C } & 3.08475 & -0.48446 & -0.22799\end{array}$

$\begin{array}{llll}\text { C } & 1.78881 & 0.03426 & -0.00474\end{array}$

$\begin{array}{llll}\mathrm{C} & 1.63167 & 1.40210 & 0.27295\end{array}$

$\begin{array}{llll}\mathrm{C} & 2.72732 & 2.24084 & 0.26056\end{array}$

$\begin{array}{llll}\text { C } & 3.98918 & 1.68927 & -0.02849\end{array}$

$\begin{array}{llll}\text { C } & 0.88338 & -1.06195 & -0.00106\end{array}$

$\begin{array}{llll}\mathrm{C} & 1.75075 & -2.32460 & -0.18817\end{array}$

$\begin{array}{llll}\mathrm{N} & 3.03036 & -1.86374 & -0.35574\end{array}$

$\begin{array}{llll}\mathrm{O} & 1.38741 & -3.46641 & -0.22167\end{array}$

$\begin{array}{llll}\text { C } & -0.48340 & -1.15034 & 0.09147\end{array}$

$\begin{array}{llll}\text { C } & -1.55985 & -0.16878 & -0.03374\end{array}$

$\begin{array}{llll}\mathrm{C} & -2.74851 & -0.83583 & 0.27881\end{array}$

$\begin{array}{llll}\mathrm{N} & -2.41696 & -2.17034 & 0.57762\end{array}$

$\begin{array}{llll}\mathrm{C} & -1.12995 & -2.38062 & 0.44332\end{array}$

$\begin{array}{llll}\text { C } & -1.65998 & 1.14148 & -0.49693\end{array}$

$\begin{array}{llll}\text { C } & -2.90918 & 1.73453 & -0.55858\end{array}$

$\begin{array}{llll}\text { C } & -4.08399 & 1.06645 & -0.18533\end{array}$

$\begin{array}{llll}\mathrm{C} & -4.00181 & -0.25945 & 0.22652\end{array}$

$\begin{array}{llll}\mathrm{H} & 5.18360 & -0.05470 & -0.44395\end{array}$

$\begin{array}{llll}\mathrm{H} & 0.66152 & 1.79455 & 0.54157\end{array}$

$\begin{array}{llll}\mathrm{C} & 2.60430 & 3.70897 & 0.55495\end{array}$

$\mathrm{H} \quad 4.84731 \quad 2.35166 \quad-0.04596$

$\begin{array}{llll}\mathrm{H} & 3.81663 & -2.47966 & -0.49084\end{array}$

$\mathrm{H} \quad-3.08367 \quad-2.87685 \quad 0.85823$

$\mathrm{H} \quad-0.67347 \quad-3.33977 \quad 0.62767$

$\mathrm{H} \quad-0.79446 \quad 1.68570 \quad-0.84381$

$\begin{array}{llll}\mathrm{H} & -4.89341 & -0.81976 & 0.47869\end{array}$

$\begin{array}{llll}\mathrm{H} & -2.98808 & 2.75257 & -0.91955\end{array}$

$\begin{array}{llll}\text { C } & -5.40518 & 1.77489 & -0.24929\end{array}$

$\mathrm{H} \quad 3.24549 \quad 3.99253 \quad 1.39015$

$\begin{array}{llll}\mathrm{H} & 2.90783 & 4.30295 & -0.30828\end{array}$

$\begin{array}{llll}\mathrm{H} & 1.58003 & 3.97639 & 0.80774\end{array}$

$\begin{array}{llll}\mathrm{H} & -6.23528 & 1.08613 & -0.10646\end{array}$

$\begin{array}{llll}\mathrm{H} & -5.46230 & 2.54047 & 0.52655\end{array}$

$\mathrm{H} \quad-5.53054 \quad 2.27340 \quad-1.21049$ 


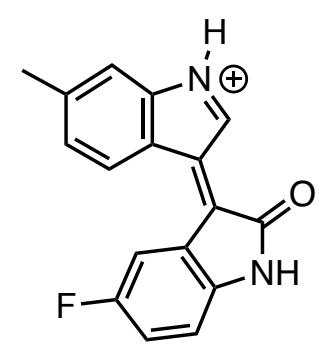

\section{Indolium 23}

$\begin{array}{lccc}\text { M06-2X/def2-TZVP Geometry } & \\ \mathrm{C} & 4.19470 & -0.40328 & 0.22786 \\ \mathrm{C} & 3.08892 & 0.42403 & 0.21013 \\ \mathrm{C} & 1.78542 & -0.07857 & 0.00000 \\ \mathrm{C} & 1.60228 & -1.43952 & -0.27519 \\ \mathrm{C} & 2.71369 & -2.24592 & -0.26473 \\ \mathrm{C} & 3.99177 & -1.75590 & -0.00325 \\ \mathrm{C} & 0.88917 & 1.03290 & 0.00833 \\ \mathrm{C} & 1.77492 & 2.28281 & 0.18923 \\ \mathrm{~N} & 3.04876 & 1.80562 & 0.34257 \\ \mathrm{O} & 1.42497 & 3.42891 & 0.22926 \\ \mathrm{C} & -0.47341 & 1.13301 & -0.07099 \\ \mathrm{C} & -1.56129 & 0.16120 & 0.04010 \\ \mathrm{C} & -2.74244 & 0.84534 & -0.26304 \\ \mathrm{~N} & -2.39604 & 2.18356 & -0.53981 \\ \mathrm{C} & -1.11075 & 2.38020 & -0.40272 \\ \mathrm{C} & -1.67648 & -1.15409 & 0.48289 \\ \mathrm{C} & -2.93288 & -1.73511 & 0.53243 \\ \mathrm{C} & -4.09896 & -1.04921 & 0.16786 \\ \mathrm{C} & -4.00135 & 0.28398 & -0.22178 \\ \mathrm{H} & 5.18914 & -0.01576 & 0.39969 \\ \mathrm{H} & 0.64279 & -1.85915 & -0.53472 \\ \mathrm{~F} & 2.57455 & -3.54450 & -0.52300 \\ \mathrm{H} & 4.82222 & -2.44983 & -0.00695 \\ \mathrm{H} & 3.84320 & 2.41228 & 0.47274 \\ \mathrm{H} & -3.05647 & 2.90107 & -0.80934 \\ \mathrm{H} & -0.64319 & 3.33730 & -0.57115 \\ \mathrm{H} & -0.81994 & -1.71423 & 0.82621 \\ \mathrm{H} & -4.88637 & 0.85840 & -0.46521 \\ \mathrm{H} & -3.02216 & -2.75794 & 0.87667 \\ \mathrm{C} & -5.42785 & -1.74379 & 0.21142 \\ \mathrm{H} & -6.25136 & -1.03571 & 0.14501 \\ \mathrm{H} & -5.51466 & -2.44293 & -0.62253 \\ \mathrm{H} & -5.53582 & -2.31707 & 1.13178\end{array}$


<smiles></smiles>

Indolium 24

$\begin{array}{lccc}\text { M06-2X/def2-TZVP Geometry } & \\ \mathrm{C} & -4.04896 & 0.27808 & 0.24584 \\ \mathrm{C} & -2.92919 & -0.53118 & 0.23587 \\ \mathrm{C} & -1.63837 & -0.01479 & -0.02302 \\ \mathrm{C} & -1.48610 & 1.33847 & -0.35825 \\ \mathrm{C} & -2.61050 & 2.12460 & -0.35564 \\ \mathrm{C} & -3.87437 & 1.62244 & -0.04419 \\ \mathrm{C} & -0.72460 & -1.10750 & 0.01084 \\ \mathrm{C} & -1.58548 & -2.36595 & 0.25772 \\ \mathrm{~N} & -2.86401 & -1.90435 & 0.42201 \\ \mathrm{O} & -1.21217 & -3.50183 & 0.33162 \\ \mathrm{C} & 0.63901 & -1.19392 & -0.08866 \\ \mathrm{C} & 1.71066 & -0.19823 & -0.01798 \\ \mathrm{C} & 2.90218 & -0.87317 & -0.31243 \\ \mathrm{~N} & 2.57644 & -2.22242 & -0.54295 \\ \mathrm{C} & 1.29541 & -2.43732 & -0.38696 \\ \mathrm{C} & 1.78229 & 1.13179 & 0.37745 \\ \mathrm{C} & 3.03316 & 1.72464 & 0.39157 \\ \mathrm{C} & 4.20457 & 1.05958 & 0.04949 \\ \mathrm{C} & 4.14927 & -0.27975 & -0.29750 \\ \mathrm{H} & -5.03243 & -0.11853 & 0.45568 \\ \mathrm{H} & -0.54152 & 1.76423 & -0.65947 \\ \mathrm{~F} & -2.50353 & 3.41207 & -0.67098 \\ \mathrm{H} & -4.71678 & 2.30191 & -0.05721 \\ \mathrm{H} & -3.64614 & -2.51827 & 0.58883 \\ \mathrm{H} & 3.24628 & -2.93696 & -0.79682 \\ \mathrm{H} & 0.84493 & -3.40772 & -0.52113 \\ \mathrm{H} & 0.93162 & 1.70643 & 0.70964 \\ \mathrm{H} & 5.04930 & -0.83242 & -0.53098 \\ \mathrm{~F} & 3.11675 & 2.99683 & 0.76153 \\ \mathrm{H} & 5.14223 & 1.59627 & 0.08514\end{array}$




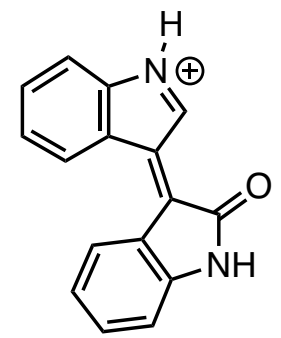

\section{Indolium 25}

$\begin{array}{lccc}\text { M06-2X/def2-TZVP Geometry } & \\ \mathrm{C} & 4.02813 & -0.61642 & 0.11484 \\ \mathrm{C} & 2.89867 & 0.17441 & 0.14213 \\ \mathrm{C} & 1.60477 & -0.36116 & -0.07193 \\ \mathrm{C} & 1.47051 & -1.71681 & -0.40678 \\ \mathrm{C} & 2.59606 & -2.51005 & -0.45043 \\ \mathrm{C} & 3.85450 & -1.96478 & -0.17191 \\ \mathrm{C} & 0.67727 & 0.71609 & -0.00685 \\ \mathrm{C} & 1.52558 & 1.98690 & 0.21018 \\ \mathrm{~N} & 2.81712 & 1.54445 & 0.33040 \\ \mathrm{O} & 1.14155 & 3.11865 & 0.29692 \\ \mathrm{C} & -0.69181 & 0.78154 & -0.06021 \\ \mathrm{C} & -1.74635 & -0.22770 & 0.05099 \\ \mathrm{C} & -2.95593 & 0.42974 & -0.20726 \\ \mathrm{~N} & -2.65617 & 1.78191 & -0.45673 \\ \mathrm{C} & -1.37162 & 2.01249 & -0.34347 \\ \mathrm{C} & -1.79748 & -1.55691 & 0.45922 \\ \mathrm{C} & -3.03209 & -2.18745 & 0.52422 \\ \mathrm{C} & -4.21197 & -1.51680 & 0.20583 \\ \mathrm{C} & -4.19420 & -0.17663 & -0.15225 \\ \mathrm{H} & 5.01171 & -0.20399 & 0.29187 \\ \mathrm{H} & 0.50675 & -2.12578 & -0.66998 \\ \mathrm{H} & 4.72481 & -2.60768 & -0.20309 \\ \mathrm{H} & 3.59457 & 2.17113 & 0.46931 \\ \mathrm{H} & -3.34307 & 2.48622 & -0.69145 \\ \mathrm{H} & -0.93854 & 2.98793 & -0.49691 \\ \mathrm{H} & -0.90863 & -2.09023 & 0.76028 \\ \mathrm{H} & -5.10656 & 0.36614 & -0.36024 \\ \mathrm{H} & -5.15663 & -2.03968 & 0.26304 \\ \mathrm{H} & -3.08079 & -3.22099 & 0.83857 \\ \mathrm{H} & 2.51319 & -3.55559 & -0.71026\end{array}$


<smiles>O=C1Nc2ccc(Cl)cc2/C1=C1\C=[NH+]c2ccccc21</smiles>

Indolium 26

$\begin{array}{lccc}\text { M06-2X/def2-TZVP Geometry } & \\ \mathrm{C} & -3.60788 & 1.03604 & 0.28085 \\ \mathrm{C} & -2.28933 & 1.44101 & 0.22726 \\ \mathrm{C} & -1.23700 & 0.51618 & 0.03811 \\ \mathrm{C} & -1.53276 & -0.83430 & -0.18470 \\ \mathrm{C} & -2.85043 & -1.23473 & -0.14255 \\ \mathrm{C} & -3.87494 & -0.31364 & 0.10582 \\ \mathrm{C} & -0.01685 & 1.25401 & 0.00123 \\ \mathrm{C} & -0.42285 & 2.73764 & 0.12620 \\ \mathrm{~N} & -1.78215 & 2.72846 & 0.30129 \\ \mathrm{O} & 0.29502 & 3.69686 & 0.11496 \\ \mathrm{C} & 1.29938 & 0.88397 & -0.07613 \\ \mathrm{C} & 1.99418 & -0.39447 & 0.09166 \\ \mathrm{C} & 3.33615 & -0.16437 & -0.23685 \\ \mathrm{~N} & 3.45969 & 1.19600 & -0.58071 \\ \mathrm{C} & 2.31748 & 1.82242 & -0.46219 \\ \mathrm{C} & 1.64898 & -1.64439 & 0.59559 \\ \mathrm{C} & 2.63043 & -2.62255 & 0.68279 \\ \mathrm{C} & 3.94521 & -2.37394 & 0.29415 \\ \mathrm{C} & 4.32791 & -1.11976 & -0.16080 \\ \mathrm{Cl} & -3.25279 & -2.88634 & -0.41193 \\ \mathrm{H} & -4.41215 & 1.74120 & 0.43799 \\ \mathrm{H} & -0.76313 & -1.54963 & -0.42995 \\ \mathrm{H} & -4.89667 & -0.66859 & 0.13752 \\ \mathrm{H} & -2.32189 & 3.57410 & 0.40186 \\ \mathrm{H} & 4.32113 & 1.63379 & -0.88057 \\ \mathrm{H} & 2.20130 & 2.87217 & -0.67990 \\ \mathrm{H} & 0.65278 & -1.85651 & 0.95284 \\ \mathrm{H} & 2.36937 & -3.59778 & 1.07022 \\ \mathrm{H} & 4.68369 & -3.15991 & 0.37126 \\ \mathrm{H} & 5.35407 & -0.90185 & -0.42495\end{array}$


<smiles>Cc1ccc2c(c1)/C(=C1/C=[NH+]c3ccc(F)cc31)C(=O)N2</smiles>

Indolium 27

$\begin{array}{lccc}\text { M06-2X/def2-TZVP Geometry } & \\ \mathrm{C} & 4.04513 & 0.35411 & 0.23575 \\ \mathrm{C} & 2.94452 & -0.48041 & 0.23738 \\ \mathrm{C} & 1.64205 & 0.00308 & -0.01880 \\ \mathrm{C} & 1.45657 & 1.34825 & -0.36196 \\ \mathrm{C} & 2.56297 & 2.16145 & -0.36983 \\ \mathrm{C} & 3.83883 & 1.69294 & -0.06234 \\ \mathrm{C} & 0.75236 & -1.11340 & 0.02449 \\ \mathrm{C} & 1.64353 & -2.34774 & 0.27704 \\ \mathrm{~N} & 2.91093 & -1.85461 & 0.43265 \\ \mathrm{O} & 1.30019 & -3.49317 & 0.36178 \\ \mathrm{C} & -0.60716 & -1.22982 & -0.07437 \\ \mathrm{C} & -1.70546 & -0.26119 & -0.02263 \\ \mathrm{C} & -2.87309 & -0.96900 & -0.32249 \\ \mathrm{~N} & -2.51234 & -2.31357 & -0.53614 \\ \mathrm{C} & -1.22933 & -2.49548 & -0.36540 \\ \mathrm{C} & -1.82378 & 1.07020 & 0.36365 \\ \mathrm{C} & -3.07669 & 1.67831 & 0.37455 \\ \mathrm{C} & -4.20901 & 0.93790 & 0.01121 \\ \mathrm{C} & -4.13177 & -0.40311 & -0.32565 \\ \mathrm{~F} & 2.42083 & 3.44556 & -0.69163 \\ \mathrm{H} & 5.03849 & -0.01862 & 0.44290 \\ \mathrm{H} & 0.49954 & 1.74855 & -0.65856 \\ \mathrm{H} & 4.66527 & 2.39133 & -0.08384 \\ \mathrm{H} & 3.70700 & -2.44893 & 0.60360 \\ \mathrm{H} & -3.16214 & -3.04653 & -0.78961 \\ \mathrm{H} & -0.75242 & -3.45509 & -0.48523 \\ \mathrm{H} & -0.96628 & 1.63580 & 0.69781 \\ \mathrm{C} & -3.22794 & 3.11812 & 0.77417 \\ \mathrm{H} & -5.17657 & 1.42390 & 0.02087 \\ \mathrm{H} & -5.01776 & -0.97713 & -0.56232 \\ \mathrm{H} & -3.97638 & 3.22413 & 1.55976 \\ \mathrm{H} & -2.28943 & 3.53264 & 1.13667 \\ \mathrm{H} & -3.55894 & 3.71721 & -0.07568\end{array}$


<smiles>Cc1ccc2c(c1)/C(=C1/C=[NH+]c3ccc(F)cc31)C(=O)N2</smiles>

Indolium 28

$\begin{array}{lccc}\text { M06-2X/def2-TZVP Geometry } & \\ \mathrm{C} & -4.04513 & 0.19431 & -0.27562 \\ \mathrm{C} & -2.92028 & -0.60488 & -0.25005 \\ \mathrm{C} & -1.63937 & -0.06652 & 0.01961 \\ \mathrm{C} & -1.51923 & 1.29453 & 0.35393 \\ \mathrm{C} & -2.63168 & 2.10847 & 0.34985 \\ \mathrm{C} & -3.87544 & 1.53985 & 0.01175 \\ \mathrm{C} & -0.71190 & -1.13949 & -0.00245 \\ \mathrm{C} & -1.54770 & -2.41424 & -0.25365 \\ \mathrm{~N} & -2.83447 & -1.97547 & -0.42890 \\ \mathrm{O} & -1.15551 & -3.54383 & -0.32193 \\ \mathrm{C} & 0.65653 & -1.20703 & 0.10860 \\ \mathrm{C} & 1.71038 & -0.19593 & 0.02240 \\ \mathrm{C} & 2.91368 & -0.84701 & 0.32440 \\ \mathrm{~N} & 2.61062 & -2.19528 & 0.57672 \\ \mathrm{C} & 1.32900 & -2.43047 & 0.42572 \\ \mathrm{C} & 1.75908 & 1.13015 & -0.39229 \\ \mathrm{C} & 2.99851 & 1.74285 & -0.41802 \\ \mathrm{C} & 4.18254 & 1.10281 & -0.06936 \\ \mathrm{C} & 4.15077 & -0.23125 & 0.29745 \\ \mathrm{C} & -2.55010 & 3.56599 & 0.70373 \\ \mathrm{H} & -5.02335 & -0.21089 & -0.49440 \\ \mathrm{H} & -0.56510 & 1.69763 & 0.66131 \\ \mathrm{H} & -4.74728 & 2.18433 & 0.00038 \\ \mathrm{H} & -3.60472 & -2.60210 & -0.60275 \\ \mathrm{H} & 3.29082 & -2.89552 & 0.84019 \\ \mathrm{H} & 0.89491 & -3.40569 & 0.57595 \\ \mathrm{H} & 0.89584 & 1.68390 & -0.72764 \\ \mathrm{~F} & 3.06055 & 3.01303 & -0.80576 \\ \mathrm{H} & 5.11073 & 1.65491 & -0.11517 \\ \mathrm{H} & 5.06060 & -0.76486 & 0.53717 \\ \mathrm{H} & -3.20388 & 3.79763 & 1.54525 \\ \mathrm{H} & -1.53509 & 3.85118 & 0.97340 \\ \mathrm{H} & -2.86526 & 4.18523 & -0.13721\end{array}$




\section{Cartesian coordinates of the reaction components relevant to secondary amine catalysis}

The following reaction components were optimized with Gaussian 16 (rev. B.01 or C.01). All other reaction components required for re-creating the statistical model have been published elsewhere. ${ }^{11}$<smiles>COc1ccc(OC)c(N2CCCC2)c1</smiles>

M06/def2-TZVP Geometry

$\begin{array}{lrrr}\mathrm{C} & 0.18680 & 1.59733 & -0.10839 \\ \mathrm{C} & 1.53384 & 1.87951 & 0.01512 \\ \mathrm{C} & 2.51974 & 0.90559 & 0.08980 \\ \mathrm{C} & 2.12906 & -0.42027 & 0.02079 \\ \mathrm{C} & 0.78243 & -0.74243 & -0.08395 \\ \mathrm{C} & -0.20364 & 0.23856 & -0.12784 \\ \mathrm{C} & -0.77757 & 2.72115 & -0.32997 \\ \mathrm{O} & 2.97839 & -1.47536 & 0.05828 \\ \mathrm{C} & 4.34693 & -1.20916 & 0.18421 \\ \mathrm{~N} & -1.54122 & -0.12678 & -0.23661 \\ \mathrm{C} & -1.90453 & -1.50326 & -0.51916 \\ \mathrm{C} & -3.41322 & -1.48775 & -0.42645 \\ \mathrm{C} & -3.62718 & -0.58587 & 0.77707 \\ \mathrm{C} & -2.58479 & 0.50517 & 0.57748 \\ \mathrm{H} & 1.83664 & 2.92242 & 0.02089 \\ \mathrm{H} & 3.55876 & 1.19363 & 0.17090 \\ \mathrm{H} & 0.53030 & -1.79411 & -0.11525 \\ \mathrm{H} & -0.23294 & 3.63997 & -0.55146 \\ \mathrm{H} & -1.43687 & 2.51269 & -1.17711 \\ \mathrm{H} & -1.41871 & 2.92586 & 0.53133 \\ \mathrm{H} & 4.57352 & -0.67158 & 1.11244 \\ \mathrm{H} & 4.85438 & -2.17253 & 0.20224 \\ \mathrm{H} & 4.72751 & -0.62390 & -0.66109 \\ \mathrm{H} & -1.51909 & -1.81166 & -1.49517 \\ \mathrm{H} & -1.49973 & -2.20341 & 0.23130 \\ \mathrm{H} & -3.84145 & -1.03559 & -1.32593 \\ \mathrm{H} & -3.84088 & -2.48368 & -0.30795 \\ \mathrm{H} & -4.63858 & -0.18638 & 0.85757 \\ \mathrm{H} & -3.41744 & -1.14619 & 1.69303 \\ \mathrm{H} & -2.17651 & 0.86640 & 1.52771 \\ \mathrm{H} & -3.02466 & 1.36699 & 0.06426\end{array}$


<smiles>CCCCCCCC=C[C](OC(C)=O)C(C)=O</smiles>

\section{Copper Intermediate 1}

M06/def2-TZVP Geometry

$\begin{array}{lrrr}\mathrm{O} & 2.05712 & -1.39825 & 0.31954 \\ \mathrm{O} & -0.09095 & 1.37604 & -0.90500 \\ \mathrm{C} & -0.60964 & -1.13883 & -0.02858 \\ \mathrm{C} & -0.44590 & 2.08297 & 0.13418 \\ \mathrm{C} & 3.08012 & -0.64095 & 0.14788 \\ \mathrm{O} & 2.89104 & 0.50040 & -0.31789 \\ \mathrm{C} & 4.42323 & -1.14772 & 0.52534 \\ \mathrm{O} & -0.17145 & 1.77798 & 1.27769 \\ \mathrm{C} & -1.25510 & 3.29634 & -0.22423 \\ \mathrm{C} & -1.70383 & -1.05970 & -0.73945 \\ \mathrm{C} & -2.87839 & -1.96859 & -0.53878 \\ \mathrm{C} & -4.11398 & -1.18375 & -0.11216 \\ \mathrm{C} & -3.94338 & -0.50365 & 1.23016 \\ \mathrm{H} & -0.32886 & -1.81870 & 0.77121 \\ \mathrm{H} & 4.49874 & -1.16699 & 1.61421 \\ \mathrm{H} & 4.55346 & -2.16973 & 0.17032 \\ \mathrm{H} & 5.20027 & -0.49993 & 0.12563 \\ \mathrm{H} & -2.25828 & 2.97328 & -0.51298 \\ \mathrm{H} & -1.32778 & 3.96298 & 0.63187 \\ \mathrm{H} & -0.82147 & 3.81518 & -1.07850 \\ \mathrm{H} & -1.81415 & -0.27912 & -1.49032 \\ \mathrm{H} & -3.07882 & -2.49821 & -1.47606 \\ \mathrm{H} & -2.63976 & -2.72662 & 0.21453 \\ \mathrm{H} & -4.34919 & -0.44087 & -0.88309 \\ \mathrm{H} & -4.96601 & -1.86963 & -0.08072 \\ \mathrm{H} & -4.85825 & 0.00268 & 1.54118 \\ \mathrm{H} & -3.68562 & -1.23136 & 2.00507 \\ \mathrm{H} & -3.14158 & 0.23891 & 1.20839 \\ \mathrm{Cu} & 0.90388 & -0.02613 & -0.28005\end{array}$<smiles>CC(=O)O[C](C=Cc1ccccc1)OC(C)=O</smiles>

\section{Copper Intermediate 2}

M06/def2-TZVP Geometry 


\begin{tabular}{|c|c|c|c|}
\hline $\mathrm{O}$ & 1.10671 & 1.66410 & -0.63756 \\
\hline $\mathrm{O}$ & 1.86197 & -1.88912 & 0.14788 \\
\hline $\mathrm{C}$ & -0.35778 & -0.44081 & 0.23641 \\
\hline $\mathrm{C}$ & 3.09099 & -1.69374 & -0.17640 \\
\hline $\mathrm{C}$ & 1.46776 & 2.35859 & 0.40909 \\
\hline $\mathrm{O}$ & 1.84940 & 1.84286 & 1.44173 \\
\hline $\mathrm{C}$ & 1.36271 & 3.84182 & 0.20996 \\
\hline $\mathrm{O}$ & 3.41928 & -0.55245 & -0.55626 \\
\hline $\mathrm{C}$ & 4.05258 & -2.81853 & -0.06765 \\
\hline $\mathrm{C}$ & -1.37309 & 0.16211 & -0.33978 \\
\hline $\mathrm{C}$ & -2.79136 & -0.15808 & -0.14724 \\
\hline $\mathrm{C}$ & -3.23445 & -1.13912 & 0.73889 \\
\hline $\mathrm{C}$ & -4.58191 & -1.39704 & 0.88360 \\
\hline $\mathrm{C}$ & -5.51516 & -0.68246 & 0.14649 \\
\hline $\mathrm{C}$ & -5.08888 & 0.29344 & -0.73610 \\
\hline $\mathrm{C}$ & -3.73771 & 0.55280 & -0.88013 \\
\hline $\mathrm{H}$ & -0.31207 & -1.25164 & 0.95582 \\
\hline $\mathrm{H}$ & 1.92149 & 4.14111 & -0.67715 \\
\hline $\mathrm{H}$ & 0.31966 & 4.11510 & 0.04334 \\
\hline $\mathrm{H}$ & 1.74320 & 4.36230 & 1.08551 \\
\hline $\mathrm{H}$ & 3.61397 & -3.72978 & -0.47352 \\
\hline $\mathrm{H}$ & 4.98023 & -2.57623 & -0.58140 \\
\hline $\mathrm{H}$ & 4.26533 & -2.99857 & 0.98786 \\
\hline $\mathrm{H}$ & -1.16847 & 0.97551 & -1.03285 \\
\hline $\mathrm{H}$ & -2.51651 & -1.70423 & 1.32252 \\
\hline $\mathrm{H}$ & -4.91077 & -2.16163 & 1.57768 \\
\hline $\mathrm{H}$ & -6.57264 & -0.88797 & 0.26301 \\
\hline $\mathrm{H}$ & -5.81052 & 0.85726 & -1.31547 \\
\hline $\mathrm{H}$ & -3.40201 & 1.31869 & -1.57156 \\
\hline $\mathrm{Cu}$ & 1.45475 & -0.07782 & -0.18666 \\
\hline \multicolumn{4}{|c|}{$\mathrm{AcO}-\mathrm{Cu}-\mathrm{OAc}$} \\
\hline
\end{tabular}

Copper Intermediate 3

\begin{tabular}{lccc}
\multicolumn{4}{l}{ M06/def2-TZVP Geometry } \\
O & -1.61586 & -1.88580 & -0.10042 \\
$\mathrm{O}$ & -1.88092 & 1.78069 & -0.66810 \\
$\mathrm{C}$ & 0.19249 & 0.28115 & -0.43337 \\
$\mathrm{C}$ & -2.23705 & 2.26664 & 0.49380 \\
$\mathrm{C}$ & -2.89854 & -1.95452 & -0.13190 \\
$\mathrm{O}$ & -3.52533 & -0.89936 & -0.35312 \\
$\mathrm{C}$ & -3.56398 & -3.25694 & 0.11269
\end{tabular}




$\begin{array}{lccc}\mathrm{O} & -2.26558 & 1.59925 & 1.50836 \\ \mathrm{C} & -2.60765 & 3.71970 & 0.44042 \\ \mathrm{C} & 0.99143 & -0.62064 & 0.09438 \\ \mathrm{C} & 2.45599 & -0.54396 & 0.13414 \\ \mathrm{C} & 3.16709 & -1.64631 & 0.60076 \\ \mathrm{C} & 4.54974 & -1.62560 & 0.65161 \\ \mathrm{C} & 5.24988 & -0.50679 & 0.24092 \\ \mathrm{C} & 4.53209 & 0.58205 & -0.21698 \\ \mathrm{C} & 3.15882 & 0.58666 & -0.27733 \\ \mathrm{~F} & 5.19482 & 1.66708 & -0.60979 \\ \mathrm{H} & 0.40803 & 1.23394 & -0.89939 \\ \mathrm{H} & -3.03141 & -4.05685 & -0.40062 \\ \mathrm{H} & -3.53072 & -3.47112 & 1.18277 \\ \mathrm{H} & -4.60302 & -3.21803 & -0.20694 \\ \mathrm{H} & -3.46549 & 3.85444 & -0.21959 \\ \mathrm{H} & -1.78500 & 4.30164 & 0.02380 \\ \mathrm{H} & -2.85103 & 4.07622 & 1.43847 \\ \mathrm{H} & 0.56478 & -1.52258 & 0.52801 \\ \mathrm{H} & 2.62345 & -2.52644 & 0.92503 \\ \mathrm{H} & 5.08908 & -2.49094 & 1.01759 \\ \mathrm{H} & 6.33101 & -0.45943 & 0.27207 \\ \mathrm{H} & 2.65362 & 1.47521 & -0.63562 \\ \mathrm{Cu} & -1.68265 & -0.02230 & -0.37538\end{array}$

$\mathrm{H}$

ÓOtBu

\section{tBuOOH}

M06/def2-TZVP Geometry

$\begin{array}{lccc}\mathrm{O} & -1.94778 & 0.11054 & -0.14555 \\ \mathrm{O} & -0.73719 & -0.02970 & -0.87526 \\ \mathrm{C} & 0.37725 & -0.00007 & 0.03080 \\ \mathrm{C} & 1.55286 & -0.13689 & -0.91401 \\ \mathrm{C} & 0.31539 & -1.17140 & 0.99036 \\ \mathrm{C} & 0.42128 & 1.32280 & 0.76656 \\ \mathrm{H} & -2.30196 & -0.78701 & -0.18912 \\ \mathrm{H} & 1.55722 & 0.67919 & -1.63878 \\ \mathrm{H} & 2.48657 & -0.10547 & -0.34922 \\ \mathrm{H} & 1.50558 & -1.08326 & -1.45637 \\ \mathrm{H} & 1.22883 & -1.21704 & 1.58748 \\ \mathrm{H} & -0.52709 & -1.07647 & 1.67722 \\ \mathrm{H} & 0.22125 & -2.11078 & 0.43921 \\ \mathrm{H} & -0.45647 & 1.44503 & 1.40170 \\ \mathrm{H} & 1.31217 & 1.37223 & 1.39728 \\ \mathrm{H} & 0.45298 & 2.15026 & 0.05490\end{array}$




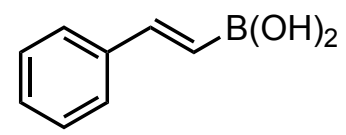

\section{Boronic acid}

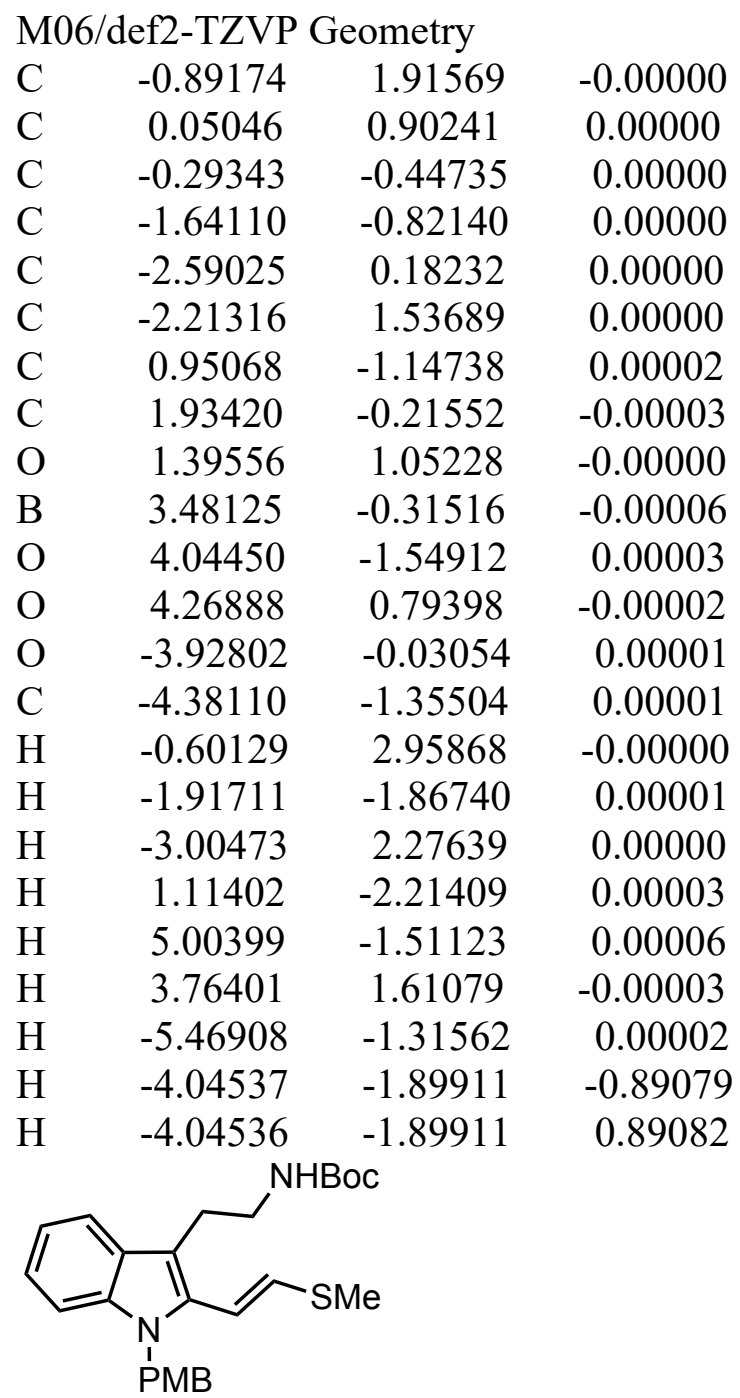

Functionalized Indole

\begin{tabular}{lrrr}
\multicolumn{4}{l}{ M06/def2-TZVP Geometry } \\
C & -1.15812 & 0.03899 & 3.00510 \\
$\mathrm{C}$ & -0.56251 & -0.82714 & 2.09712 \\
$\mathrm{C}$ & -1.28564 & -1.85221 & 1.45310 \\
$\mathrm{C}$ & -2.64501 & -2.00013 & 1.73745 \\
$\mathrm{C}$ & -3.23875 & -1.14641 & 2.64226 \\
$\mathrm{C}$ & -2.50111 & -0.13673 & 3.27123 \\
$\mathrm{C}$ & -0.37120 & -2.53409 & 0.60309 \\
$\mathrm{C}$ & 0.85189 & -1.91515 & 0.74100 \\
$\mathrm{~N}$ & 0.73824 & -0.88648 & 1.66597 \\
$\mathrm{C}$ & 1.76012 & 0.01402 & 2.13101
\end{tabular}




\begin{tabular}{|c|c|c|c|}
\hline $\mathrm{C}$ & 1.78377 & 1.33598 & 1.40849 \\
\hline $\mathrm{C}$ & 2.05245 & -2.27104 & 0.02236 \\
\hline $\mathrm{C}$ & -0.70569 & -3.59619 & -0.38235 \\
\hline $\mathrm{C}$ & -0.98351 & -3.02982 & -1.77431 \\
\hline $\mathrm{C}$ & 3.07692 & -1.48060 & -0.32263 \\
\hline S & 4.51109 & -1.90176 & -1.20374 \\
\hline $\mathrm{C}$ & 4.26500 & -3.65081 & -1.52598 \\
\hline $\mathrm{N}$ & -2.11487 & -2.13875 & -1.79629 \\
\hline $\mathrm{C}$ & -1.99111 & -0.78634 & -1.70949 \\
\hline $\mathrm{O}$ & -0.95402 & -0.17710 & -1.81770 \\
\hline $\mathrm{O}$ & -3.20686 & -0.25228 & -1.52163 \\
\hline $\mathrm{C}$ & -3.38660 & 1.16917 & -1.28340 \\
\hline $\mathrm{C}$ & -4.87369 & 1.27276 & -1.01528 \\
\hline $\mathrm{C}$ & -3.01283 & 1.95739 & -2.52187 \\
\hline $\mathrm{C}$ & -2.60983 & 1.61434 & -0.06136 \\
\hline $\mathrm{C}$ & 1.17820 & 1.50599 & 0.17639 \\
\hline $\mathrm{C}$ & 1.22442 & 2.72520 & -0.48578 \\
\hline $\mathrm{C}$ & 1.88764 & 3.79835 & 0.09001 \\
\hline $\mathrm{C}$ & 2.50245 & 3.63689 & 1.33007 \\
\hline $\mathrm{C}$ & 2.44648 & 2.42212 & 1.97467 \\
\hline $\mathrm{O}$ & 1.98606 & 5.02703 & -0.46449 \\
\hline $\mathrm{C}$ & 1.36851 & 5.23943 & -1.70493 \\
\hline $\mathrm{H}$ & -0.59681 & 0.83799 & 3.47658 \\
\hline $\mathrm{H}$ & -3.22718 & -2.77961 & 1.25616 \\
\hline $\mathrm{H}$ & -4.29247 & -1.25435 & 2.87210 \\
\hline $\mathrm{H}$ & -2.99579 & 0.52544 & 3.97201 \\
\hline $\mathrm{H}$ & 1.60289 & 0.18266 & 3.20208 \\
\hline $\mathrm{H}$ & 2.72566 & -0.49382 & 2.04878 \\
\hline $\mathrm{H}$ & 2.06475 & -3.30769 & -0.30322 \\
\hline $\mathrm{H}$ & 0.10265 & -4.33140 & -0.46457 \\
\hline $\mathrm{H}$ & -1.58530 & -4.15484 & -0.04110 \\
\hline $\mathrm{H}$ & -0.11978 & -2.45957 & -2.12373 \\
\hline $\mathrm{H}$ & -1.15289 & -3.84388 & -2.48663 \\
\hline $\mathrm{H}$ & 3.07829 & -0.41984 & -0.08987 \\
\hline $\mathrm{H}$ & 4.18060 & -4.21525 & -0.59582 \\
\hline $\mathrm{H}$ & 3.37973 & -3.81699 & -2.14208 \\
\hline $\mathrm{H}$ & 5.14457 & -3.99068 & -2.07026 \\
\hline $\mathrm{H}$ & -3.02577 & -2.50720 & -1.57540 \\
\hline $\mathrm{H}$ & -5.13913 & 0.68685 & -0.13210 \\
\hline $\mathrm{H}$ & -5.15107 & 2.31396 & -0.83921 \\
\hline $\mathrm{H}$ & -5.44545 & 0.89864 & -1.86708 \\
\hline $\mathrm{H}$ & -1.94887 & 1.87156 & -2.73845 \\
\hline $\mathrm{H}$ & -3.57934 & 1.60139 & -3.38568 \\
\hline $\mathrm{H}$ & -3.25589 & 3.01162 & -2.36651 \\
\hline $\mathrm{H}$ & -1.53542 & 1.61819 & -0.23689 \\
\hline $\mathrm{H}$ & -2.91754 & 2.62821 & 0.20678 \\
\hline
\end{tabular}




$\begin{array}{lrrr}\mathrm{H} & -2.82309 & 0.95887 & 0.78784 \\ \mathrm{H} & 0.64258 & 0.68598 & -0.29417 \\ \mathrm{H} & 0.73437 & 2.81348 & -1.44730 \\ \mathrm{H} & 3.01129 & 4.48708 & 1.76880 \\ \mathrm{H} & 2.92206 & 2.31142 & 2.94537 \\ \mathrm{H} & 1.55171 & 6.27878 & -1.97220 \\ \mathrm{H} & 1.78817 & 4.59255 & -2.48364 \\ \mathrm{H} & 0.28655 & 5.07007 & -1.65381\end{array}$

$1^{1}$

Alkynyl aldehyde

M06-2X/def2-TZVP Geometry

$\begin{array}{llll}\text { C } & 1.86229 & -0.13118 & -0.00001\end{array}$

$\begin{array}{llll}\mathrm{C} & 0.68397 & 0.08542 & 0.00002\end{array}$

$\begin{array}{llll}\mathrm{C} & -0.73390 & 0.40975 & 0.00001\end{array}$

$\begin{array}{llll}\mathrm{O} & -1.60259 & -0.41640 & -0.00001\end{array}$

$\mathrm{H} \quad 2.90620 \quad-0.34096 \quad-0.00003$

$\mathrm{H} \quad-0.95964 \quad 1.48816 \quad-0.00004$<smiles>CC(C)CC1NC(Cc2cccc3ccccc23)C(=O)N1C</smiles>

\section{Amine Catalyst}

M06-2X/def2-TZVP Geometry

$\begin{array}{lrrr}\mathrm{N} & -0.98521 & 0.33006 & -1.46793 \\ \mathrm{C} & -2.08330 & 0.79368 & -0.62193 \\ \mathrm{~N} & -2.56022 & -0.42349 & 0.04258 \\ \mathrm{C} & -2.05750 & -1.55001 & -0.51853 \\ \mathrm{C} & -1.10015 & -1.11575 & -1.61908 \\ \mathrm{C} & 0.22326 & -1.88469 & -1.56197 \\ \mathrm{C} & -1.64560 & 1.93236 & 0.32881 \\ \mathrm{C} & -2.87363 & 2.63537 & 0.91673 \\ \mathrm{C} & -0.86896 & 2.97112 & -0.49083 \\ \mathrm{C} & -0.76852 & 1.39744 & 1.46154 \\ \mathrm{C} & 1.04758 & -1.54866 & -0.34380 \\ \mathrm{C} & 0.79659 & -2.21172 & 0.83134 \\ \mathrm{C} & 1.49617 & -1.90605 & 2.01678 \\ \mathrm{C} & 2.45314 & -0.93379 & 2.01818\end{array}$




$\begin{array}{cccc}\mathrm{C} & 2.74849 & -0.21874 & 0.83331 \\ \mathrm{C} & 2.04153 & -0.52076 & -0.36384 \\ \mathrm{C} & 3.72404 & 0.80807 & 0.82576 \\ \mathrm{C} & 3.98890 & 1.51994 & -0.30845 \\ \mathrm{C} & 3.28416 & 1.23343 & -1.49627 \\ \mathrm{C} & 2.34262 & 0.24242 & -1.52412 \\ \mathrm{O} & -2.34837 & -2.69294 & -0.23220 \\ \mathrm{C} & -3.72411 & -0.48511 & 0.89299 \\ \mathrm{H} & -0.11109 & 0.54656 & -1.00605 \\ \mathrm{H} & -2.89695 & 1.18119 & -1.25400 \\ \mathrm{H} & -1.59491 & -1.36058 & -2.56611 \\ \mathrm{H} & -0.04374 & -2.94219 & -1.53287 \\ \mathrm{H} & 0.78153 & -1.71985 & -2.48204 \\ \mathrm{H} & -2.55290 & 3.49901 & 1.50189 \\ \mathrm{H} & -3.45032 & 1.99113 & 1.57751 \\ \mathrm{H} & -3.53433 & 2.99619 & 0.12504 \\ \mathrm{H} & -0.68363 & 3.85345 & 0.12397 \\ \mathrm{H} & 0.09718 & 2.59673 & -0.83158 \\ \mathrm{H} & -1.43849 & 3.28061 & -1.36993 \\ \mathrm{H} & -0.41957 & 2.22821 & 2.07789 \\ \mathrm{H} & -1.31931 & 0.70681 & 2.10161 \\ \mathrm{H} & 0.11373 & 0.86964 & 1.09197 \\ \mathrm{H} & 0.03413 & -2.98183 & 0.84394 \\ \mathrm{H} & 1.26834 & -2.45129 & 2.92343 \\ \mathrm{H} & 2.99810 & -0.69079 & 2.92241 \\ \mathrm{H} & 4.25468 & 1.02079 & 1.74623 \\ \mathrm{H} & 4.73412 & 2.30449 & -0.30049 \\ \mathrm{H} & 3.49165 & 1.80277 & -2.39299 \\ \mathrm{H} & 1.80774 & 0.05308 & -2.44479 \\ \mathrm{H} & -4.51465 & 0.15709 & 0.49739 \\ \mathrm{H} & -4.06572 & -1.51884 & 0.90814 \\ \mathrm{H} & -3.49881 & -0.17626 & 1.91476\end{array}$


Models Used

Secondary amine model as described in ref. 11

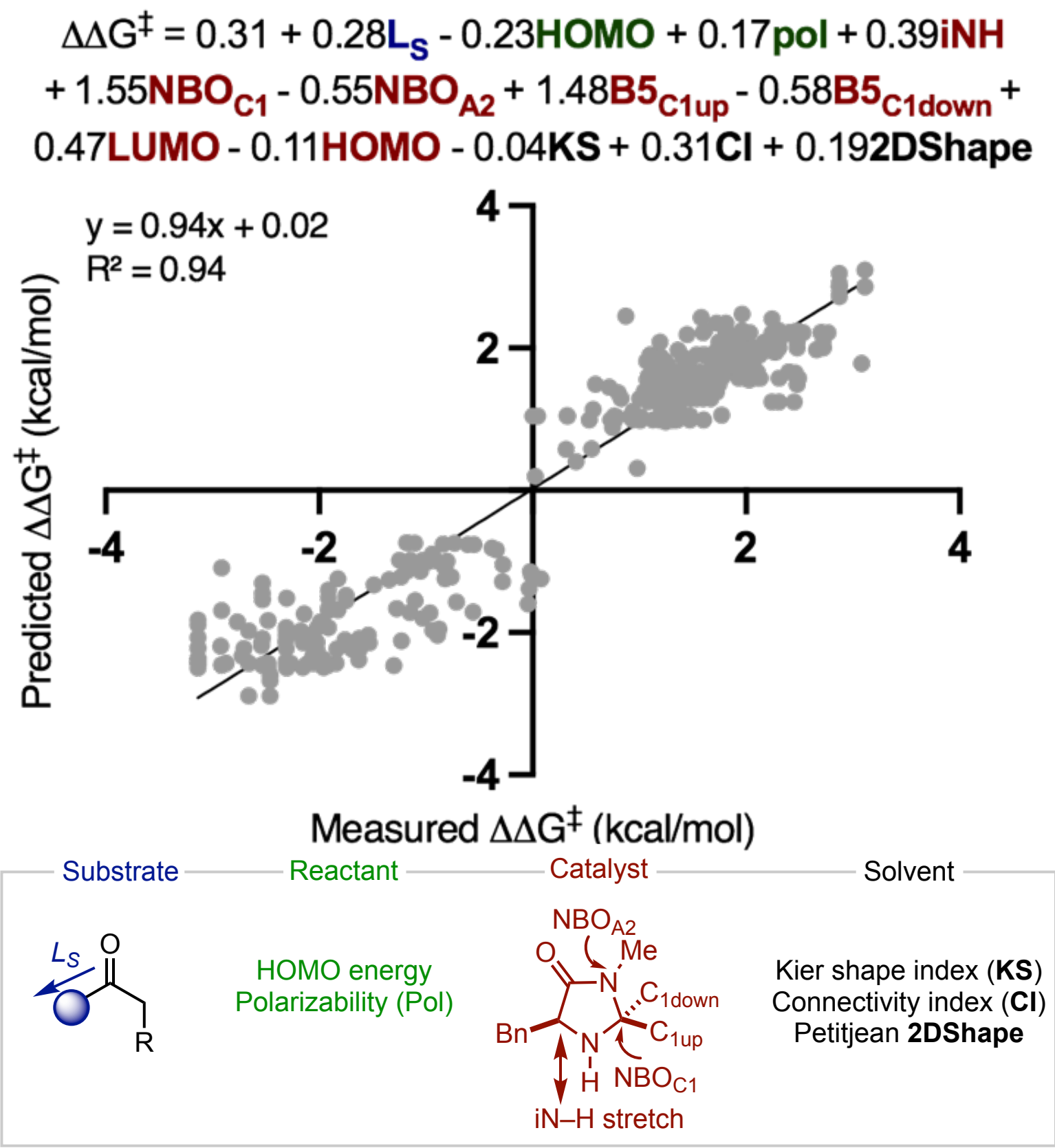

$\mathrm{KS}=$ kier alpha modified shape index

$\mathrm{CI}=$ averaged valence connectivity index chi-5

2DShape $=$ Petitjean 2D shape 


\section{More details on parameters collected for using the secondary amine model}

From the ground state DFT optimized aldehyde structure, Sterimol L values were calculated using the Bondi radii. HOMO energies and polarizabilities were calculated and collected for the reacting structure. IR stretching frequencies and intensities, NBO charges, HOMO and LUMO energies, and Sterimol B5 values were calculated and collected for the catalyst structure. Solvent topological and connectivity descriptors were acquired from DFT optimized geometries and calculated with the molecular descriptors application available in Maestro using default settings. 


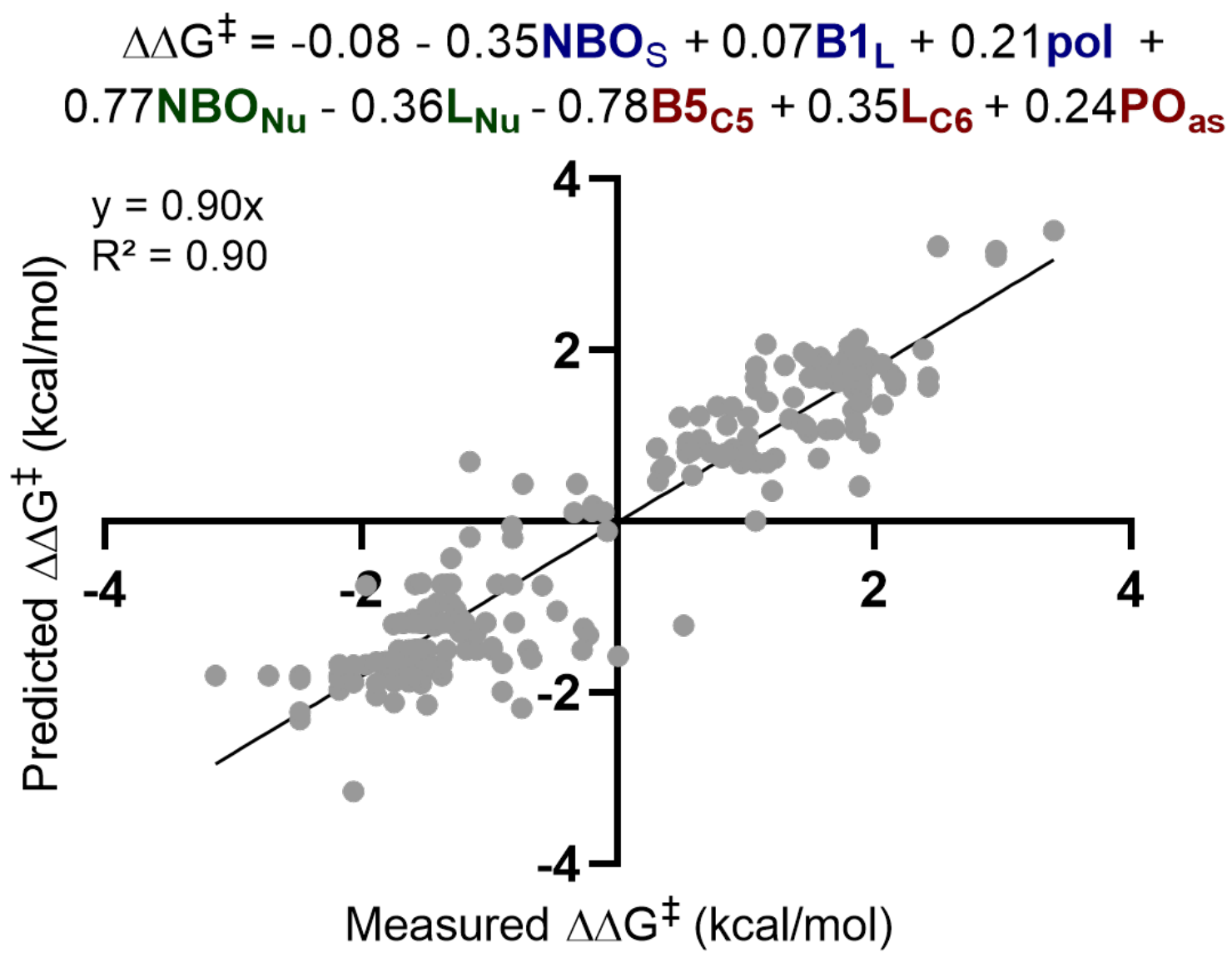

Iminium

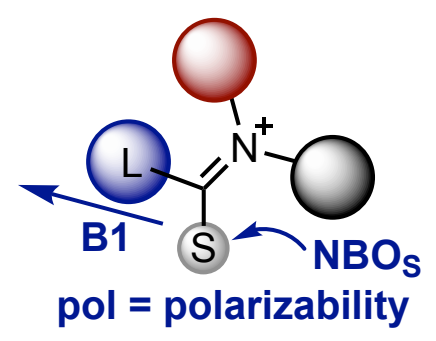

Nucleophile

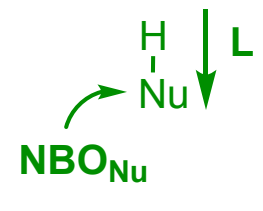

Catalyst

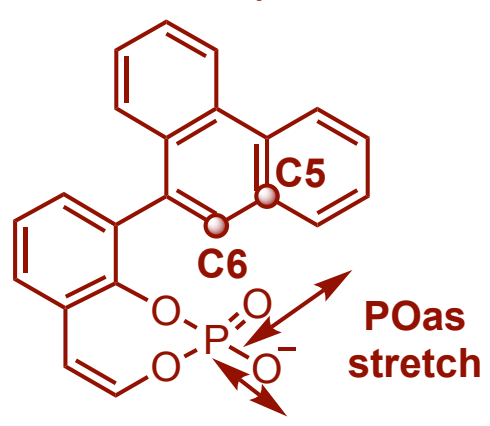




\section{More details on parameters collected for using the chiral phosphate model}

From the ground state DFT optimized iminium structure NBO charges and Sterimol values of the necessary substituent in addition to polarizability were calculated and collected. Sterimol $\mathrm{L}$ value of the entire nucleophile unit and the NBO charge of the nucleophilic site were necessary for prediction. The vibration term POas (the asymmetric $\mathrm{P}-\mathrm{O}$ stretching frequency) and Sterimol values from the $\mathrm{C} 5$ and $\mathrm{C} 6$ positions were calculated and collected from simple model phosphoric acid structures.

\section{Out-of-sample Predictions}

The accuracy of the out-of-sample predictions was determined by error in both enantioselectivity (e.e. values) and the average absolute $\Delta \Delta \mathrm{G}^{\ddagger}$ error $\left(\Delta \Delta \mathrm{G}^{\ddagger}\right.$ error $=$ absolute (model measured experimentally measured/ no. of examples). The workflow for e.e. prediction consists of locating the ground state of the targeted reaction variable by DFT computation and collecting the required parameters. The parameters are then submitted to the desired model which provides a predicted $\Delta \Delta \mathrm{G}^{\ddagger}$ value that can be converted to an e.e. value. The process is described pictorially below for a selected substrate catalyzed by a chiral phosphate.
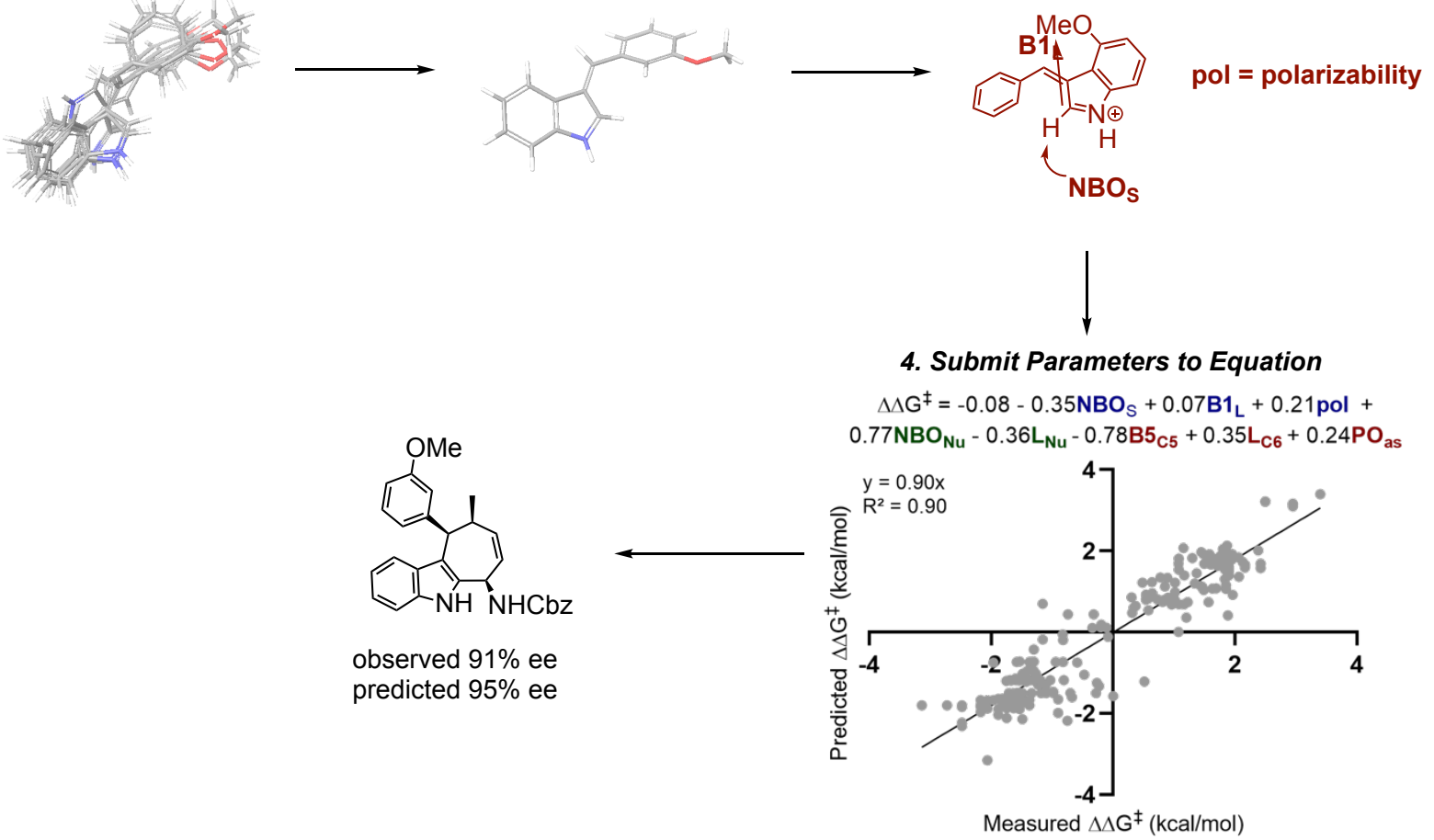

All parameters and numerical out-of-sample prediction results can be found in the supplementary excel sheet. 
A key aspect of utilizing previously published models is to keep the computational methods consistent. To this end, we have quantified the differences between parameters collected from Gaussian 09 optimized geometries and those from Gaussian 16 (using the same method). As can been seen from the accompanying spreadsheet, the parameters did not change significantly so the matching of computational software's is not critical for prediction but is preferable.

\section{Data curation}

Relevant data were manually extracted from the following sources:

\section{Out-of-sample CPA}

(1) Gelis, C.; Levitre, G.; Merad, J.; Retailleau, P.; Neuville, L.; Masson, G. Highly Diastereo- and Enantioselective Synthesis of Cyclohepta[ $b$ ]Indoles by Chiral-Phosphoric-Acid-Catalyzed $(4+3)$ Cycloaddition. Angew. Chem. Int. Ed. 2018, 57, 12121-12125.

(2) Guo, C.; Song, J.; Huang, J.-Z.; Chen, P.-H.; Luo, S.-W.; Gong, L.-Z. Core-Structure-Oriented Asymmetric Organocatalytic Substitution of 3-Hydroxyoxindoles: Application in the Enantioselective Total Synthesis of (+)-Folicanthine. Angew. Chem. Int. Ed. 2012, 51, 1046-1050.

(3) Song, J.; Guo, C.; Adele, A.; Yin, H.; Gong, L. Enantioselective Organocatalytic Construction of Hexahydropyrroloindole by Means of A-Alkylation of Aldehydes Leading to the Total Synthesis of (+)-Gliocladin C. Chem. Eur. J. 2013, 19, 3319-3323.

\section{Out-of-sample synergistic/secondary amine}

(1) Jones, S. B.; Simmons, B.; MacMillan, D. W. C. Nine-Step Enantioselective Total Synthesis of (+)-Minfiensine. J. Am. Chem. Soc. 2009, 131, 13606-13607.

(2) Paras, N. A.; Simmons, B.; MacMillan, D. W. C. A Process for the Rapid Removal of Dialkylamino-Substituents from Aromatic Rings. Application to the Expedient Synthesis of (R)Tolterodine. Tetrahedron 2009, 65, 3232-3238.

(3) Reiter, M.; Torssell, S.; Lee, S.; MacMillan, D. W. C. The Organocatalytic Three-Step Total Synthesis of (+)-Frondosin B. Chem. Sci. 2010, 1, 37-42.

(4) Stevens, J. M.; MacMillan, D. W. C. Enantioselective $\alpha$-alkenylation of aldehydes with boronic acids via the synergistic combination of Copper (II) and amine catalysis. J. Am. Chem. Soc. 2013, $135,11756-11759$.

Predicting different catalyst chemotypes and pathways to access the same intermediate (1) Wang, X.; List, B. Asymmetric Counteranion-Directed Catalysis for the Epoxidation of Enals. Angew. Chem. Int. Ed. 2008, 47, 1119-1122.

(2) Marigo, M.; Franzén, J.; Poulsen, T. B.; Zhuang, W.; Jørgensen, K. A. Asymmetric Organocatalytic Epoxidation of $\alpha, \beta$-Unsaturated Aldehydes with Hydrogen Peroxide. J. Am. Chem. Soc. 2005, 127, 6964-6965.

(3) Bertelsen, S.; Dinér, P.; Johansen, R. L.; Jørgensen, K. A. Asymmetric Organocatalytic $\beta$ Hydroxylation of $\alpha, \beta$-Unsaturated Aldehydes. J. Am. Chem. Soc. 2007, 129, 1536-1537. 


\section{Principal Component Analysis (PCA)}

Principle component analysis was performed to illustrate structural differences between reaction space included in the training set and that observed in out-of-sample predictions. Data points for the following plots can be found in the accompanying excel spreadsheet.

\section{Chiral Phosphate Catalysis}

As extrapolation to diverse catalysts was not the aim of this study, only nucleophile and electrophile parameters were included in this analysis. Of the five principal components, $74 \%$ of the observed variance can be captured by PC1 and PC2, and 88\% of the observed variance is captured by the first three principal components.

\begin{tabular}{lll}
\hline PC & \% Var & Cum \%Var \\
\hline 1 & 41.2 & 41.2 \\
\hline 2 & 33.0 & 74.2 \\
\hline 3 & 14.1 & 88.3 \\
\hline
\end{tabular}

PCA Plot of First Two Principal Components of Chiral Phosphate Model

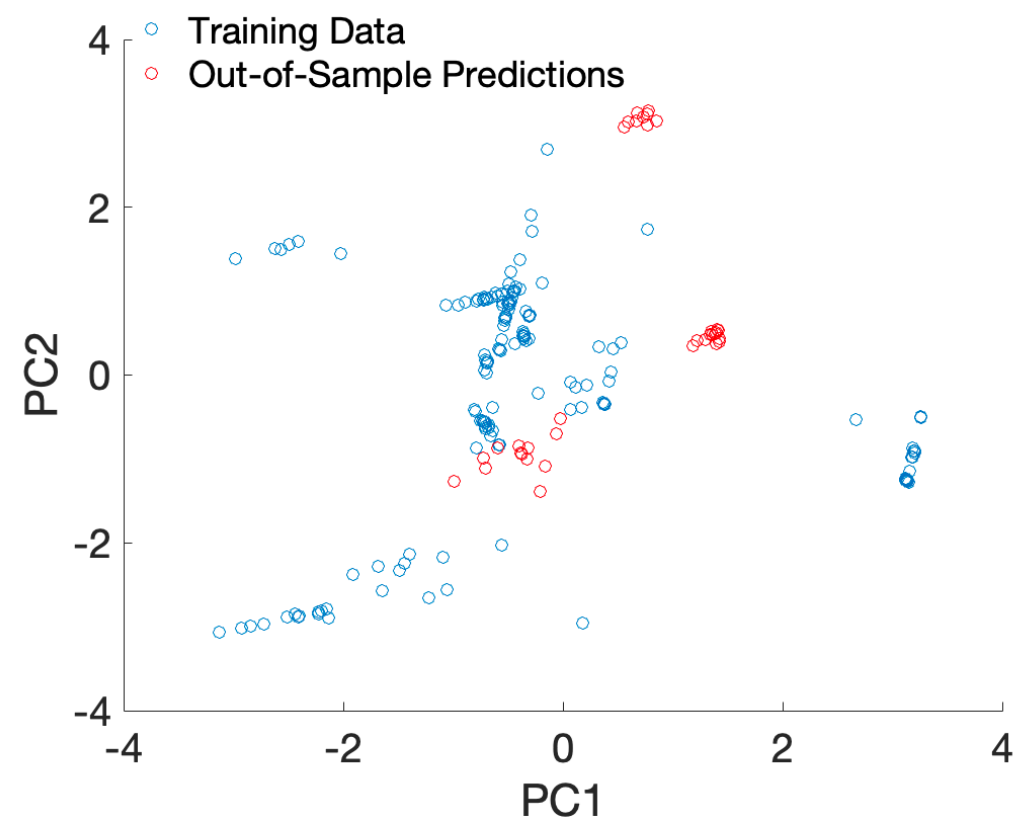


PCA Plot of First Three Principal Components of Chiral Phosphate Model

- Training Data

- Out-of-Sample Predictions

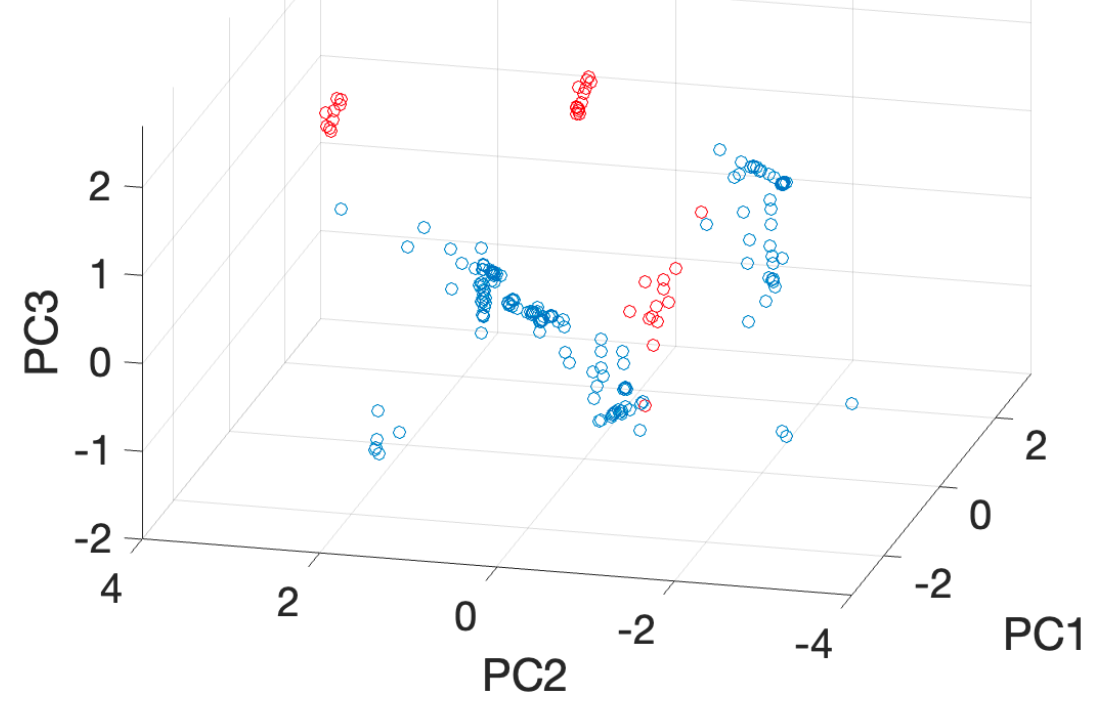




\section{Secondary Amine Catalysis}

Parameters describing varying reaction components between train and test sets were included in this analysis (catalyst, substrate and reactant). Of the 10 principal components, $52 \%$ of the observed variance is captured by $\mathrm{PC} 1$ and $\mathrm{PC} 2$, and $67 \%$ of the observed variance is captured by the first three principal components.

\begin{tabular}{lll}
\hline PC & \% Var & Cum \%Var \\
\hline 1 & 31.6 & 31.6 \\
\hline 2 & 20.3 & 51.9 \\
\hline 3 & 15.2 & 67.1 \\
\hline
\end{tabular}

PCA Plot of First Two Principal Components of Secondary Amine Model

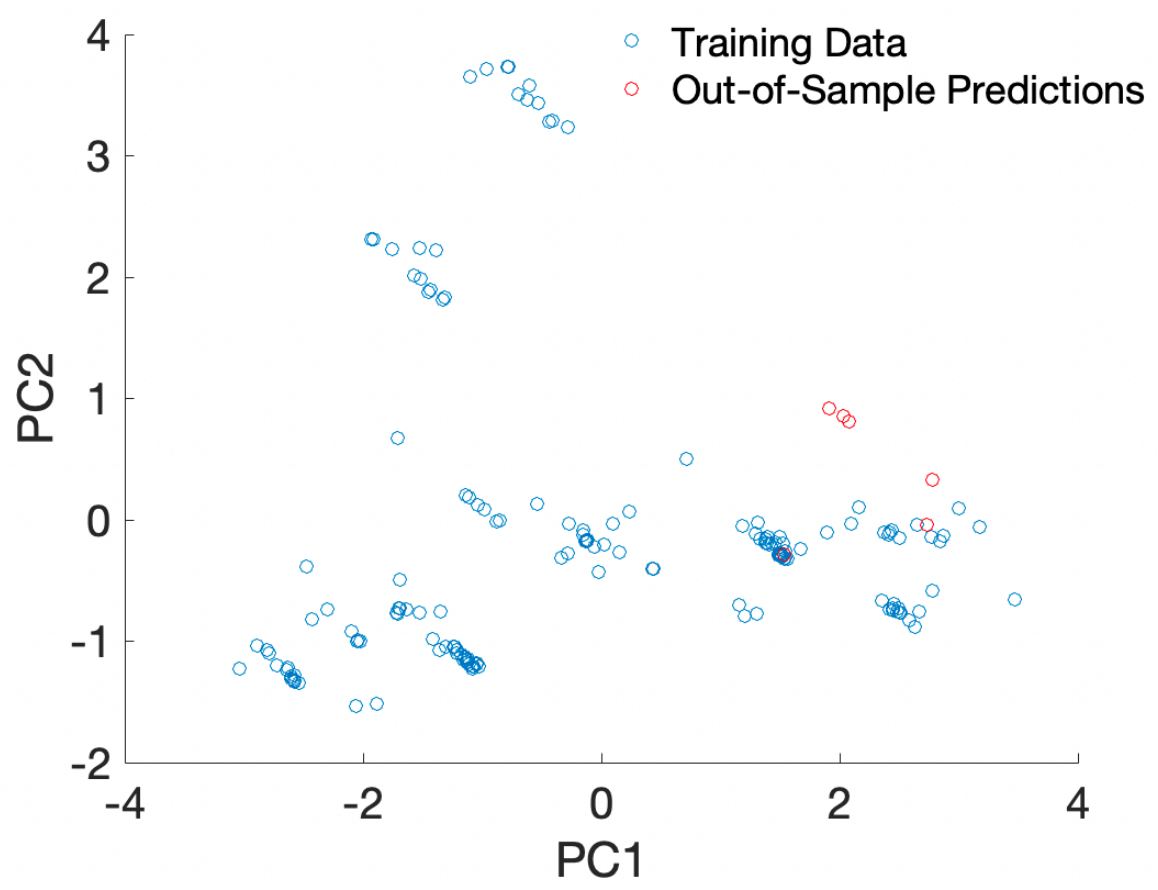


PCA Plot of First Three Principal Components of Secondary Amine Model

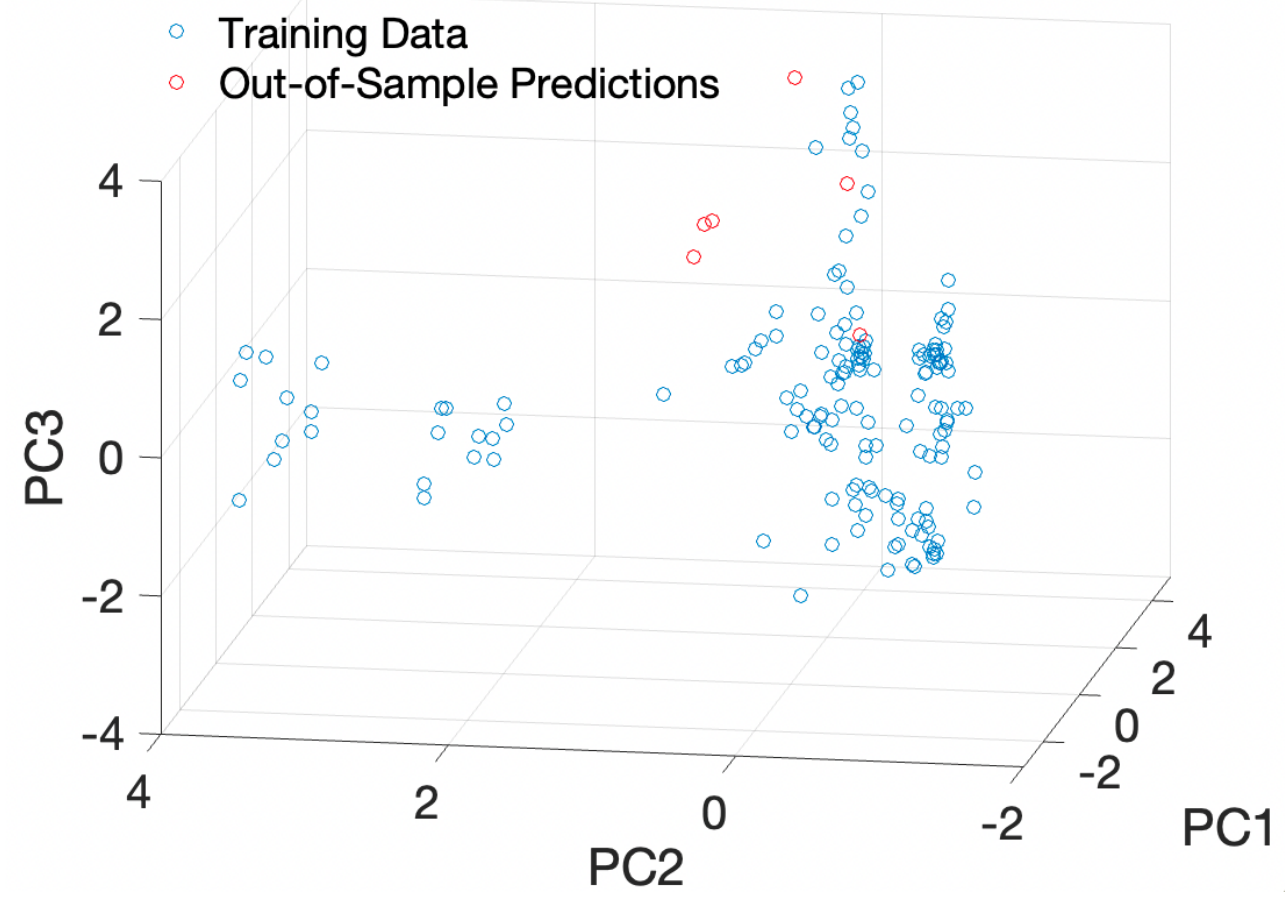




\section{Analysis of Variance (ANOVA)}

\section{CPA Training Set $N$-way ANOVA}

\begin{tabular}{lccccc} 
& & \multicolumn{5}{c}{ Analysis of Variance } \\
\hline Source & Sum Sq. & d.f. & Mean Sq. & F & Prob>F \\
\hline NBO(RS) & 15.795 & 1 & 15.7952 & 59.78 & 0 \\
B1(RL) & 0.265 & 1 & 0.2654 & 1 & 0.3172 \\
Polarizability & 6.54 & 1 & 6.5399 & 24.75 & 0 \\
Nu & 39.089 & 1 & 39.0887 & 147.94 & 0 \\
L & 13.64 & 1 & 13.6403 & 51.63 & 0 \\
B5 & 65.021 & 1 & 65.0208 & 246.09 & 0 \\
L6 & 18.755 & 1 & 18.7551 & 70.98 & 0 \\
nP0as & 7.85 & 1 & 7.8504 & 29.71 & 0 \\
Error & 64.204 & 243 & 0.2642 & & \\
Total & 652.655 & 251 & & &
\end{tabular}

Constrained (Type III) sums of squares.

CPA out-of-sample Figure $3 C$

\begin{tabular}{llllll} 
& & \multicolumn{5}{c}{ Analysis of Variance } \\
\hline Source & Sum Sq. & d.f. & Mean Sq. & F & Prob>F \\
\hline NB0(RS) & 0.1966 & 1 & 0.1966 & 1.99 & 0.1917 \\
B1(RL) & 0.11389 & 1 & 0.11389 & 1.15 & 0.3106 \\
Polarizability & 0.05158 & 1 & 0.05158 & 0.52 & 0.488 \\
\# Nu & 0 & 0 & 0 & 0 & NaN \\
\# L & 0 & 0 & 0 & 0 & NaN \\
\# B5 & 0 & 0 & 0 & 0 & NaN \\
\# L6 & 0 & 0 & 0 & 0 & NaN \\
\# nP0as & 0 & 0 & 0 & 0 & NaN \\
Error & 0.88782 & 9 & 0.09865 & & \\
Total & 1.18551 & 12 & & &
\end{tabular}


CPA out-of-sample Figure $3 A$ and $B$

\section{Analysis of Variance}

\begin{tabular}{lccccc}
\hline Source & Sum Sq. & d.f. & Mean Sq. & F & Prob>F \\
\hline NB0(RS) & 0.00003 & 1 & 0.00003 & 0 & 0.9713 \\
B1(RL) & 0.10651 & 1 & 0.10651 & 4.35 & 0.0523 \\
Polarizability & 0.14492 & 1 & 0.14492 & 5.92 & 0.0262 \\
Nu & 0.04952 & 1 & 0.04952 & 2.02 & 0.1729 \\
L & 0.04691 & 1 & 0.04691 & 1.92 & 0.184 \\
B5 & 0.16897 & 1 & 0.16897 & 6.91 & 0.0176 \\
L6 & 0.00264 & 1 & 0.00264 & 0.11 & 0.7466 \\
nP0as & 0.00054 & 1 & 0.00054 & 0.02 & 0.8831 \\
Error & 0.41582 & 17 & 0.02446 & & \\
Total & 3.23703 & 25 & & &
\end{tabular}

Constrained (Type III) sums of squares.

As out-of-sample reactions in Figure $3 \mathrm{~A}$ and $\mathrm{B}$ were mechanistically similar, they were grouped together for ANOVA analysis.

$\underline{\text { Secondary Amine Training Set N-Way ANOVA }}$

\begin{tabular}{lrrrrl} 
& & \multicolumn{5}{c}{ Analysis of Variance } \\
\hline Source & Sum Sq. & d.f. & Mean Sq. & F & Prob>F \\
\hline LC1small & 20.83 & 1 & 20.8316 & 89.34 & 0 \\
HOM0 & 12.7 & 1 & 12.7049 & 54.49 & 0 \\
Polarizabillity & 9.15 & 1 & 9.1477 & 39.23 & 0 \\
iNH & 15.21 & 1 & 15.205 & 65.21 & 0 \\
C1 & 27.74 & 1 & 27.7405 & 118.97 & 0 \\
C2 & 4.11 & 1 & 4.114 & 17.64 & 0 \\
B5C1up & 93.76 & 1 & 93.7618 & 402.11 & 0 \\
B5C1down & 41.27 & 1 & 41.2691 & 176.99 & 0 \\
LUM0 & 11.08 & 1 & 11.082 & 47.53 & 0 \\
HOM0 & 2.42 & 1 & 2.4243 & 10.4 & 0.0014 \\
Kier SI & 0.16 & 1 & 0.1607 & 0.69 & 0.4068 \\
CI & 16.62 & 1 & 16.6163 & 71.26 & 0 \\
2Dshape & 6.16 & 1 & 6.1641 & 26.44 & 0 \\
Error & 102.13 & 438 & 0.2332 & & \\
Total & 1625.96 & 451 & & &
\end{tabular}

Constrained (Type III) sums of squares. 
Histogram of Observed Error

Histograms of observed error values are shown to better illustrate the number of accurate predictions. The mean average error value reported in the text is slightly inflated due to a small number of poor predictions.

CPA out-of-sample Reaction One (Figure 3A)

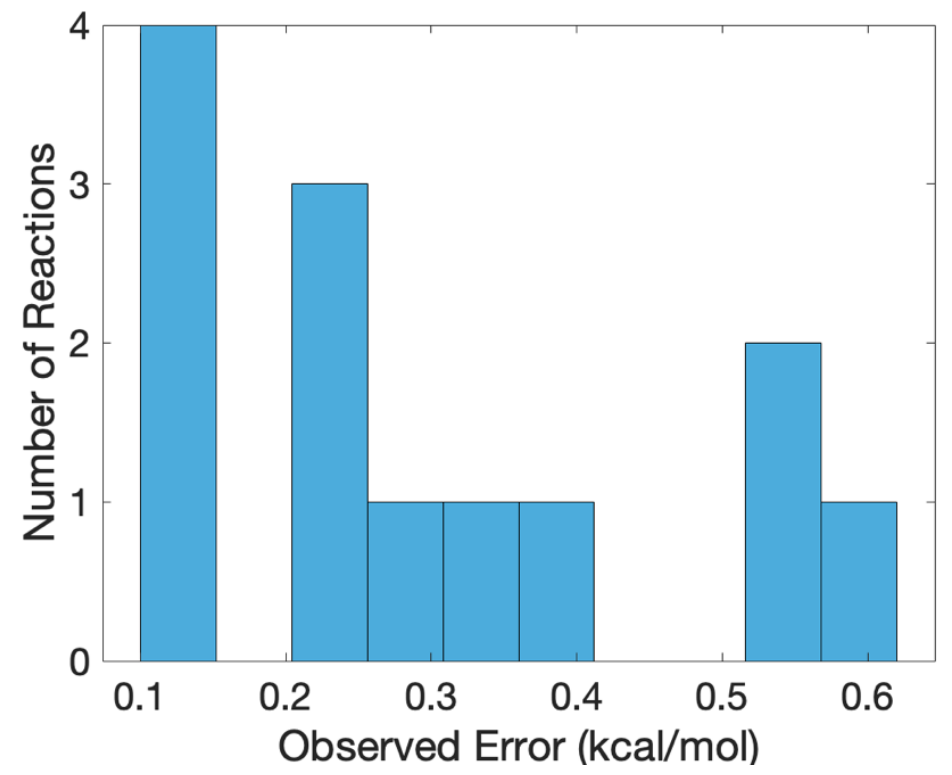

CPA out-of-sample Reaction Two (Figure 3B)

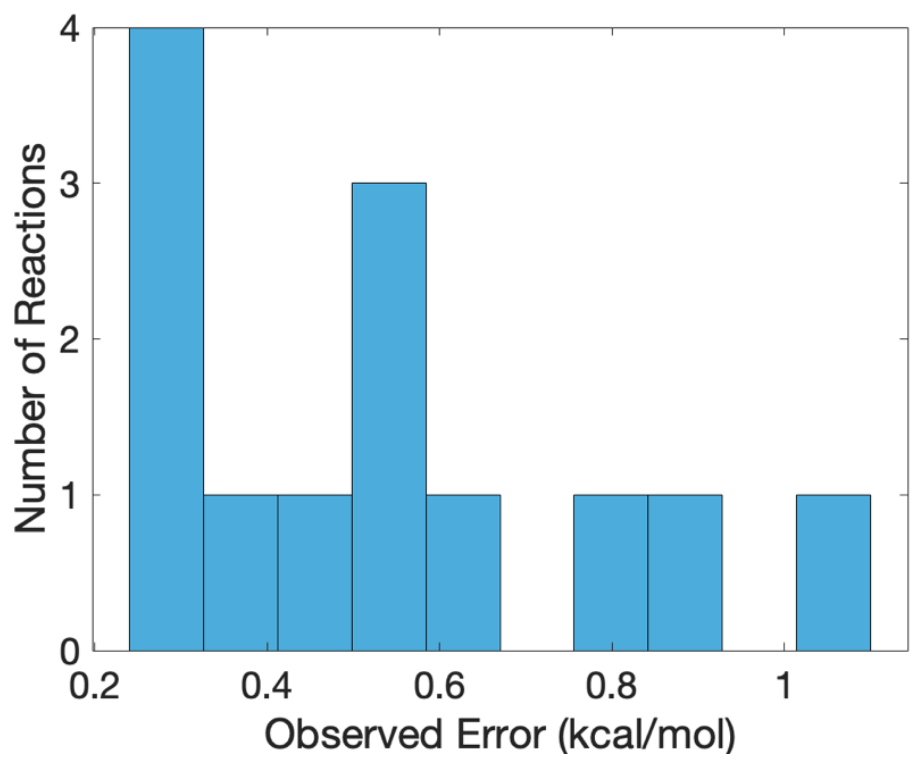


CPA out-of-sample Reaction Three (Figure 3C)

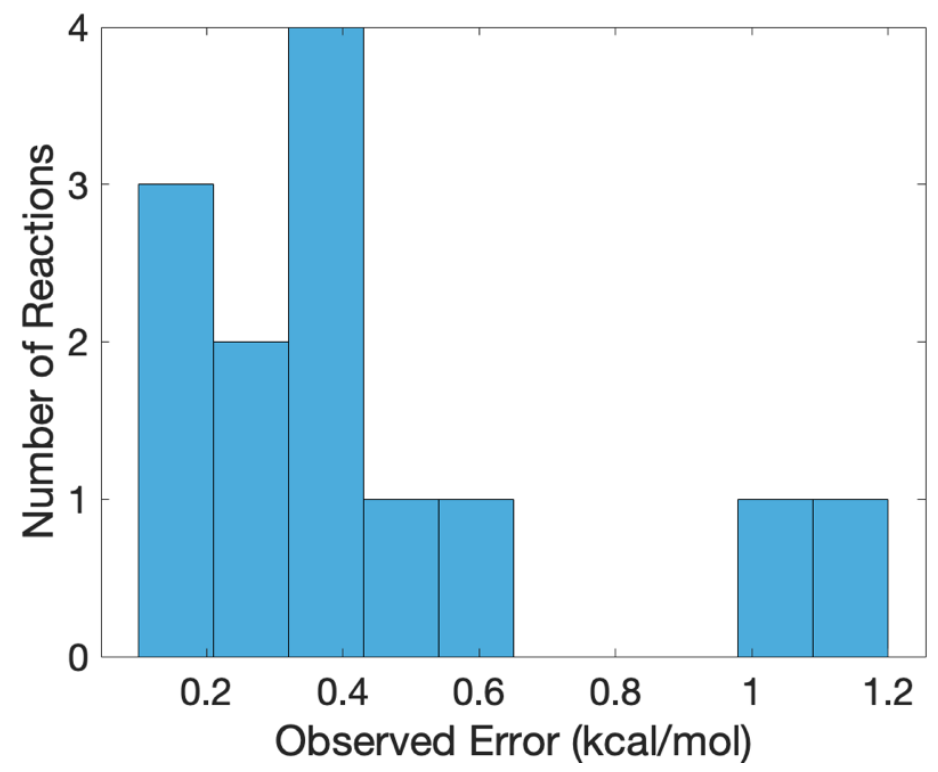




\section{References}

(1) Zhao, Y.; Truhlar, D. G. The M06 suite of density functionals for main group thermochemistry, thermochemical kinetics, noncovalent interactions, excited states, and transition elements: two new functionals and systematic testing of four M06-class functionals and 12 other functionals. Theor. Chem. Acc. 2008, 120, 215-241.

(2) Weigend, F.; Ahlrichs, R. Balanced basis sets of split valence, triple zeta valence and quadruple zeta valence quality for $\mathrm{H}$ to $\mathrm{Rn}$ : Design and assessment of accuracy. Phys. Chem. Chem. Phys. 2005, 7, 3297-3305.

(3) Frisch, M. J. et al. Gaussian 16, Revision C.01, Gaussian, Inc.: Wallingford, CT 2016.

(4) Ermanis, K.; Parkes, K. E. B.; Agback, T.; Goodman, J. M. Expanding DP4: application to drug compounds and automation. Org. Biomol. Chem., 2016, 14, 3943-3949.

(5) Glendening, E. D. et al. NBO 6.0. (Theoretical Chemistry Institute, University of Wisconsin, Madison, WI, 2013), http://nbo6.chem.wisc.edu/.

(6) Piou, T. et al. Correlating Reactivity and Selectivity to Cyclopentadienyl Ligand Properties in Rh(III)-Catalyzed C-H Activation Reactions: An Experimental and Computational Study. J. Am. Chem. Soc. 2017, 139, 1296-1310.

(7) MacroModel, version 11.7, Schrödinger, LLC, New York, NY, 2017.

(8) Lu, C. et al. OPLS4: Improving Force Field Accuracy on Challenging Regimes of Chemical Space. J. Chem. Theory Comput. 2021, 17 (7), 4291-4300

(9) MATLAB and Statistics Toolbox Release R2018b, The MathWorks, Inc., Natick, Massachusetts, United States.

(10) Shoja, A. et al. Comprehensive Stereochemical Models for Selectivity Prediction in Diverse Chiral Phosphate-Catalyzed Reaction Space. ACS Catal. 2021, 11, 11897-11905.

(11) Kuang, Y.; Reid, J. P. Transferable Selectivity Profiles Enable Prediction in Synergistic Catalyst Space. ChemRxiv 2021, DOI: 10.26434/chemrxiv-2021-q5n18 